\title{
A PROBLEM WITH AN OBSTACLE THAT GOES OUT TO THE BOUNDARY OF THE DOMAIN FOR A CLASS OF QUADRATIC FUNCTIONALS ON $\mathbb{R}^{N}$
}

\author{
A. A. ARKHIPOVA
}

Dedicated to Vasiliu Mikhaulovich Babich

\begin{abstract}
A variational problem with obstacle is studied for a quadratic functional defined on vector-valued functions $u: \Omega \rightarrow \mathbb{R}^{N}, N>1$. It is assumed that the nondiagonal matrix that determines the quadratic form of the integrand depends on the solution and is "split". The role of the obstacle is played by a closed (possibly, noncompact) set $\mathcal{K}$ in $\mathbb{R}^{N}$ or a smooth hypersurface $S$. It is assumed that $u(x) \in \mathcal{K}$ or $u(x) \in S$ a.e. on $\Omega$. This is a generalization of a scalar problem with an obstacle that goes out to the boundary of the domain. It is proved that the solutions of the variational problems in question are partially smooth in $\bar{\Omega}$ and that the singular set $\Sigma$ of the solution satisfies $H_{n-2}(\Sigma)=0$.
\end{abstract}

\section{INTRODUCTION}

Let $\mathcal{K}$ be a domain in $\mathbb{R}^{N}, N>1$, with $\mathcal{C}^{2}$-smooth boundary $\partial \mathcal{K}$, and let $\mathcal{K}=\dot{\mathcal{K}} \cup \partial \mathcal{K}$. The set $\mathcal{K}$ may be noncompact in $\mathbb{R}^{N}$.

Let $\Omega$ be a bounded domain in $\mathbb{R}^{n}, n \geq 2$, with sufficiently smooth boundary $\partial \Omega$. Consider the variational problem

$$
F[u]=\int_{\Omega}\left(\left(A(x, u) u_{x}, u_{x}\right)+a(x)|u|^{2}+f(x) u\right) d x \rightarrow \min _{W_{\mathcal{K}}}
$$

where

$$
W_{\mathcal{K}}=\left\{u \in W_{2}^{1}\left(\Omega ; \mathbb{R}^{N}\right), u(x) \in \mathcal{K} \text { for a.e. } x \in \Omega\right\} .
$$

Here $A(x, u)$ is a positive definite symmetric matrix of size $n N \times n N, a$ and $f$ are known functions, $a(x) \geq a_{0}>0$, and the function $u: \Omega \mapsto \mathbb{R}^{N}, N>1$, has the form $u=\left(u^{1}, \ldots, u^{N}\right), u_{x}=\left\{u_{x_{\alpha}}^{k}\right\}_{\alpha \leq n}^{k \leq N}$.

Note that the functions $u$ belonging to $W_{\mathcal{K}}$ have the following property: $u(x) \in \mathcal{K}$ a.e. on $\partial \Omega$. Thus, we consider a problem with an obstacle expanding up to the boundary.

In this paper, we study the regularity of functions at which the minimum in problem $(1),(2)$ is attained. Certainly, we could modify the problem slightly in order to ensure the existence of a nontrivial solution. Specifically, this can be done by assuming that $a=f=0$ and by imposing a Dirichlet condition on a part of the boundary $\partial \Omega$.

In the present paper, we also consider an obstacle expanding up to the boundary and determined by the condition $u(\Omega) \subset S$, where $S$ is a smooth hypersurface in $\mathbb{R}^{N}$. More

2010 Mathematics Subject Classification. Primary 35J20.

Key words and phrases. Variational problem, quadratic functional, nondiagonal matrix, Signorini condition.

Supported by RFBR (grant no. 09-01-00729) and by the grant NSH-4210.2010.1 for support of leading scientific schools. 
precisely, for the functional (1), we study the regularity of a solution of the variational problem

$$
\begin{gathered}
F[u] \rightarrow \min _{W_{S}} \\
W_{S}=\left\{u \in W_{2}^{1}\left(\Omega ; \mathbb{R}^{N}\right), u(x) \in S \text { for a.e. } x \in \Omega\right\} .
\end{gathered}
$$

The main condition on the matrix $A$ is its split structure:

$$
A_{k l}^{\alpha \beta}(x, u)=a^{\alpha \beta}(x) b_{k l}(x, u), \quad \alpha, \beta \leq n, \quad k, l \leq N,
$$

where $a^{\alpha \beta}$ and $b_{k l}$ are symmetric positive definite matrices on $\mathbb{R}^{n}$ and $\mathbb{R}^{N}$, respectively.

It should be noted that, in the case where $b_{k l}=b_{k l}(u), a=f=0,\left.u\right|_{\gamma}=\phi, \gamma \subset \partial \Omega(\phi$ is a given function with $\phi(\gamma) \subset \mathcal{K}$ or $\phi(\gamma) \subset \mathcal{S}$ ), the split structure (5) makes it possible to treat the variational problems in question as problems about harmonic mappings (written in local coordinates) in the situation where the entire image is covered by a single chart and the Signorini condition is fulfilled on $\partial \Omega \backslash \gamma$.

The first results about the partial regularity of functions giving a local minimum for functionals such as (11) were obtained by Giusti and Giaquinta in 1]. In that paper it was shown that a free local minimum $u$ of class $W_{2, \text { loc }}^{1}(\Omega)$ (i.e., in an obstacle-free problem) is Hölder continuous on some open set $\Omega_{0} \subset \Omega$, and that $H_{n-2}(\Sigma)=0$ for the closed set $\Sigma=\Omega \backslash \Omega_{0}$. By a well-known result of Morrey, $\Sigma=\varnothing$ in the two-dimensional case. The authors of [1, 2] also considered quadratic functionals with a split matrix (5). In this situation, in [2] it was proved that for a bounded local minimum of the functional (11), (2) we have the following estimate of the singular set: $\operatorname{dim}_{H} \Sigma \leq n-3$, and in dimension 3 , the set $\Sigma$ is either empty or consists of isolated points. A similar result on the regularity of minimums near the boundary under the Dirichlet condition was obtained by Jost and Meier [3].

The obstacle problem for quadratic functionals of the form (1), (5) and under the Dirichlet boundary condition has been studied by Hildebrandt, Widman, Fuchs, Duzaar, Wiegner and other authors (see [4, 5, 6, 7, 8, 9, 10, 11, 12, 13, 14] and the references therein). Various obstacles of the type $u(\Omega) \subset \mathcal{K}$ have been treated, where $\mathcal{K} \subset \mathbb{R}^{N}$. In particular, it was shown that $\operatorname{dim}_{H} \Sigma \leq n-3$, and $\Sigma$ may only consist of isolated points if $n=3$ and $\mathcal{K}$ is a compact subset of $\mathbb{R}^{N}$ with $\mathcal{C}^{3}$-smooth boundary $\partial \mathcal{K}$ (see [7).

Various restrictions on $\mathcal{K}$ of a geometric nature have been stated under which the solution of the variational problem with obstacle is smooth on $\bar{\Omega}$ (see [13, 14, 4, 11]). In the author's paper [15, partial regularity up to the boundary was proved for functions that provide the minimal value for the functional (11), (5) in the case of noncompact $\mathcal{K}$ with $\partial \mathcal{K} \in \mathcal{C}^{2}$. In the same paper, obstacles of the form $u(\Omega) \subset S$, where $S$ is a noncompact hypersurface in $\mathbb{R}^{N}$, were considered. In both cases, it was proved that $H_{n-2}(\Sigma)=0$ for the possible singular set $\Sigma$.

It should be noted that in all papers mentioned above the regularly near the boundary for solutions of the variational problems was studied under the Dirichlet boundary condition.

Regularity for a problem with obstacles on the boundary $\left(u(\partial \Omega) \subset \mathcal{K} \subset \mathbb{R}^{N}, N>1\right)$, i.e., the Signorini problem, has been studied since the 1970s. The regularity of solutions of a scalar Signorini problem has been explored in more detail; see [16, 17, 18, 19, 20, 21] and the references therein. In particular, under various conditions on the elliptic operator of the problem, it was proved by Caffarelli [17, Kinderlehrer [19], and Ural'tseva 20] that the solutions of variational inequalities are $\mathcal{C}^{1, \alpha}$-smooth. The optimal smoothness $u \in \mathcal{C}^{1,1 / 2}(\bar{\Omega})$ for the solution of the Signorini problem was established recently by Athanasopoulos and Caffarelli 22 . 
Under various restrictions on a convex set $\mathcal{K} \subset \mathbb{R}^{N}, N>1$, for linear operators, in [23, 24, 25] it was proved that the solutions of the Signorini problem belong to $W_{2}^{2}\left(\Omega ; \mathbb{R}^{N}\right)$. Shumann [26] proved that, in the case of linear operators of elasticity theory, the solutions of the Signorini problem in the half-space are $\mathcal{C}^{1, \alpha}$-smooth. The joint papers [27, 28, 29] by the author and Ural'tseva were devoted to the regularity problem for solutions of variational inequalities with diagonal linear and strongly nonlinear elliptic operators under convex restrictions fixed at the boundary.

In [30, the author proved partial regularity for the solutions of Signorini-type variational problems with functionals of the form (11), (5). More precisely, smoothness was studied for the minimizers of such functionals on the sets $V_{\mathcal{K}}=\left\{u \in W_{2}^{1}\left(\Omega ; \mathbb{R}^{N}\right), u(x) \in\right.$ $\mathcal{K}$ for a.e. $x \in \partial \Omega\}$ and $V_{S}=\left\{u \in W_{2}^{1}\left(\Omega ; \mathbb{R}^{N}\right), u(x) \in S\right.$ for a.e. $\left.x \in \partial \Omega\right\}$, where $\mathcal{K}$ and $S$ are as in (2) and (4).

In the present paper, we consider the situation in which the obstacle $\mathcal{K} \subset \mathbb{R}^{N}$ expands up to the boundary of $\Omega$. We shall prove a partial regularity result for the solutions of problems (11), (2) and (3), (4) similar to the results of [30]. This will be done by the local penalty method.

We shall pay more attention to problem (11), (2). In $\S 1$, we present the main assumptions about the data of that problem and state the principal result (Theorem 1). In $\S 2$, the local statement of the problem in the half-ball is presented and the local penalty method is described. In $\S 3$, a monotonicity inequality is obtained for the local normalized energy of penalty problems. In $\S 4$, we show that the solutions of penalty problems are smooth near the point at which the normalized energy integral for the solution of the original problem is small. $\S 5$ is devoted to an estimate, uniform in the penalty parameter, for the maximum of the modulus of the gradient for the solutions of penalty problems. Here the split structure (5) of the matrix $A(x, u)$ is used substantially. It enables us to apply certain methods pertinent to scalar boundary-value problems. In $\S 6$, we present a Hölder estimate for the first derivatives of the solution of the variational problem in question (in the local setting). It should be noted that only the limit function obeys this estimate, and no uniformity with respect to the parameter of penalty problems is proved. Also in that section, we finish to prove the main result, Theorem 1 . The last section, $\S 7$, is devoted to the analysis of problem (3), (4). Here Theorem 4 is proved, which is the main partial regularity result for the solution of that problem.

The following notation is adopted in the paper:

$$
\begin{aligned}
& B_{R}\left(x^{0}\right)=\left\{x \in \mathbb{R}^{n}:\left|x-x^{0}\right|<R\right\}, \\
& S_{R}\left(x^{0}\right)=\left\{x \in \mathbb{R}^{n}:\left|x-x_{0}\right|=R\right\}, \\
& S_{R}^{+}\left(x^{0}\right)=S_{R} \cap\left\{x_{n}>x_{n}^{0}\right\}, \\
& \Gamma_{R}\left(x^{0}\right)=B_{R}\left(x^{0}\right) \cap\left\{x_{n}=x_{n}^{0}\right\}, \\
& \Omega_{R}\left(x^{0}\right)=\Omega \cap B_{R}\left(x^{0}\right) ;
\end{aligned}
$$

we write $B_{R}, B_{R}^{+}, \Gamma_{R}, S_{R}^{+}$if $x^{0}=0$;

$|A|=$ meas $_{n} A$ is the Lebesgue measure of a subset $A$ of $\mathbb{R}^{n}, \omega_{n}=$ meas $_{n} B_{1}(0)$;

$$
\begin{aligned}
f_{\Omega_{r}\left(x^{0}\right)} g d x & =\frac{1}{\left|\Omega_{r}\right|} \int_{\Omega_{r}\left(x^{0}\right)} g d x, \quad f_{\Omega_{r}} g d x=\frac{1}{r^{n-2}} \int_{\Omega_{r}} g d x, \\
f_{\Gamma_{r}} f d \Gamma & =\frac{1}{r^{n-2}} \int_{\Gamma_{r}} f d \Gamma ; \quad v_{x_{\alpha}}=\frac{\partial v}{\partial x_{\alpha}}, \quad[g(x, u)]_{x_{\alpha}}^{\prime}=g_{x_{\alpha}}+g_{u} u_{x_{\alpha}} .
\end{aligned}
$$

For short, we write $u \in \mathbf{B}(\Omega)$ instead of $u \in \mathbf{B}\left(\Omega ; \mathbb{R}^{N}\right)$. Various constants depending on the data of the problem will be denoted by $c, c_{i}$. The dependence of constants on the penalty parameter $\epsilon$ is indicated separately each time. 


\section{§1. Statement of the main Results}

We list the main assumptions about the problem data.

$\left[\mathbb{A}_{\mathcal{K}}\right]$ Let $\mathcal{K} \subset \mathbb{R}^{N}$ be the closure of a domain with $\mathcal{C}^{2}$-smooth boundary $\partial \mathcal{K}$. There exist numbers $\delta_{0}$ and $M>0$ such that a $\mathcal{C}^{2}$-smooth distance function $d(u)=\operatorname{dist}(u, \partial \mathcal{K})$ is defined in the neighborhood $U_{2 \delta_{0}}(\mathcal{K})$, and

$$
\sup _{u \in U_{2 \delta_{0}}(\mathcal{K})}\left\|d^{\prime \prime}(u)\right\| \leq M .
$$

$\left[\mathcal{A}_{S}\right] S$ is a $\mathcal{C}^{2}$-smooth hypersurface without boundary in $\mathbb{R}^{N}$; there exist numbers $\delta_{0}$ and $M$ such that the distance function is defined in the two-sided neighborhood $U_{2 \delta_{0}}(S)=U_{2 \delta_{0}}^{(1)}(S) \cup S \cup U_{\delta_{0}}^{(2)}(S), d \in \mathcal{C}^{2}\left(\overline{U_{2 \delta_{0}}^{i}(S)}\right), i=1,2$, and

$$
\frac{\sup }{U_{2 \delta_{0}}^{i}(S)}\left|d^{\prime \prime}(u)\right| \leq M, \quad i=1,2 .
$$

$\left[\mathbb{A}_{1}\right]$ The matrix $a(x)=\left\{a^{\alpha \beta}(x)\right\}^{\alpha, \beta \leq n}$ is defined and $\mathcal{C}^{1}$-smooth on $\bar{\Omega}, a^{\alpha \beta}(x)=$ $a^{\beta \alpha}(x)$, and

$$
(a(x) \xi, \xi) \geq \nu_{1}|\xi|^{2}, \quad \xi \in \mathbb{R}^{N}, \quad x \in \bar{\Omega}, \quad \nu_{1}=\text { const }>0 .
$$

$\left[\mathbb{A}_{2}\right]$ The matrix $b(x, u)=\left\{b_{k l}(x, u)\right\}_{k, l \leq N}$ is defined and $\mathcal{C}^{1}$-smooth on $\bar{\Omega} \times \mathbb{R}^{N}$,

$$
\begin{aligned}
& \sup _{\Omega \times \mathbb{R}^{N}}\left\{|b(x, u)|+\left|b_{x}^{\prime}(x, u)\right|+\left|b_{u}^{\prime}(x, u)\right|\right\} \leq \mu, \quad b_{k l}(x, u)=b_{l k}(x, u), \\
& (b(x, u) \eta, \eta) \geq \nu_{2}|\eta|^{2}, \quad \eta \in \mathbb{R}^{N}, \quad(x, u) \in \bar{\Omega} \times \mathbb{R}^{N}, \quad \nu_{2}=\text { const }>0 .
\end{aligned}
$$

$\left[\mathbb{A}_{3}\right] a \in L_{q / 2}(\Omega), f \in L_{q}(\Omega), q>n, a(x) \geq a_{0}>0$. $\Omega$ is a bounded domain in $\mathbb{R}^{n}$, $n \geq 2$, with $\mathcal{C}^{2}$-smooth boundary $\partial \Omega$.

Remark 1 . If the set $\mathcal{K}$ or the surface $S$ is compact in $\mathbb{R}^{N}$, it suffices to assume that they are merely $\mathcal{C}^{2}$-smooth. For a noncompact hypersurface $S$, condition $\left[\mathcal{A}_{S}\right]$ ensures that this surface is not glued at infinity and its principal curvatures are uniformly bounded.

The main results of the paper are described by the following theorems.

Theorem 1. Suppose that conditions $\left[\mathbb{A}_{\mathcal{K}}\right]$ and $\left[\mathbb{A}_{1}\right]-\left[\mathbb{A}_{3}\right]$ are satisfied and that the minimum of the functional (1), (5) on the set $W_{\mathcal{K}}$ defined by $(2)$ is attained at the function $u \in W_{\mathcal{K}}$. Then there exists $\beta \in(0,1 / 2)$ such that $u \in \mathcal{C}^{1, \beta}\left(\Omega_{0}\right)$, where $\Omega_{0}$ is relatively open in $\bar{\Omega}$, and the closed singular set $\Sigma=\bar{\Omega} \backslash \Omega_{0}$ admits the estimate $H_{n-2}(\Sigma)=0$.

Theorem 2. Suppose that conditions $\left[\mathbb{A}_{S}\right]$ and $\left[\mathbb{A}_{1}\right]-\left[\mathbb{A}_{3}\right]$ are satisfied and that the minimum of the functional (1), (5) on the set $W_{S}$ defined by (4) is attained at a function $u$. Then the claim of Theorem 1 holds true.

\section{§2. Local PENALTy Method}

Consider problem (1), (2), (5). Suppose that the functional (1), (5) attains its minimum on the set $(2)$ at the function $u \in W_{\mathcal{K}}$. Fixing $\theta_{0}$ and $R_{0}$ (which are arbitrary for the moment), we define the set

$$
\Sigma_{\theta_{0}, R_{0}}=\bigcap_{r \leq R_{0}}\left\{x^{0} \in \bar{\Omega}: f_{\Omega_{r}\left(x^{0}\right)} e[u] d x \geq \theta_{0}^{2}\right\}, \quad e[u]=\frac{1}{2}\left(A(x, u) u_{x}, u_{x}\right) .
$$

Put

$$
\Omega_{0}=\bar{\Omega} \backslash \Sigma_{\theta_{0}, R_{0}} .
$$

We want to show that, for $\theta_{0}$ and $R_{0}$ sufficiently small, $\Omega_{0}$ is a set of regular points for $u$. 
By the definition (9), we have

$$
f_{\Omega_{R_{1}}\left(x^{0}\right)} e[u](x) d x<\theta_{0}^{2}
$$

for some $R_{1}=R_{1}\left(x^{0}\right) \leq R_{0}$, provided that $x^{0} \in \Omega_{0}$.

In order to prove Theorem 1 , first we verify the following claim.

Theorem 3. Suppose that conditions $\left[\mathbb{A}_{\mathcal{K}}\right],\left[\mathbb{A}_{1}\right]-\left[\mathbb{A}_{3}\right]$ are fulfilled. There exist constants $\theta_{0}$ and $R_{0}$ such that $\Omega_{0}$ is relatively open in $\bar{\Omega}$ and $u \in \mathcal{C}^{1, \beta}\left(\Omega_{0}\right)$ with some $\beta \in(0,1 / 2)$. The constants $\theta_{0}$ and $R_{0}$ depend on the parameters in conditions $\left[\mathbb{A}_{\mathcal{K}}\right],\left[\mathbb{A}_{1}\right]-\left[\mathbb{A}_{3}\right]$.

We prove Theorem 3 in the case where $x^{0} \in \partial \Omega \cap \Omega_{0}$.

We fix the parameters $\theta_{0}$ and $R_{0}$, arbitrary for the moment, and consider a point $x^{0} \in$ $\partial \Omega \cap \Omega_{0}$. At this point, the solution $u$ of problem (10), (2), (5) obeys condition (10). At a neighborhood of $x^{0}$, we rectify the boundary with the help of a $\mathcal{C}^{2}$-smooth transformation $y=y(x)$ such that $B_{R_{2}}^{+}(0) \subset y\left(\Omega_{R_{1}}\left(x^{0}\right)\right), \Gamma_{R_{2}}(0) \subset y\left(\partial \Omega \cap B_{R_{1}}(0)\right)$ for some $R_{2}>$ 0 , where $R_{1}$ is taken from (10). (Clearly, this rectification procedure imposes some restrictions on the smallness of $R_{0}=R_{0}(\partial \Omega)$.)

We do not change the notation for independent variables and consider the local model problem

$$
\begin{aligned}
\hat{F}\left[w ; B_{R_{2}}^{+}\right] & =\frac{1}{2} \int_{B_{R_{2}}^{+}(0)}\left[\left(\widehat{A}(x, w) w_{x}, w_{x}\right)+\widehat{a}|w|^{2}+\widehat{f} w\right] d x \mapsto \min _{W_{\mathcal{K}}^{+}} \\
W_{\mathcal{K}}^{+} & =\left\{v \in W_{2}^{1}\left(B_{R_{2}}^{+} ; \mathbb{R}^{N}\right),\left.v\right|_{B_{R_{2}}^{+}} \in \mathcal{K}, v-\left.u\right|_{S_{R_{2}}^{+}}=0\right\} .
\end{aligned}
$$

Here $\hat{A}, \hat{a}$, and $\hat{f}$ possess the same properties as in the definition (1), and the function $u$, when calculated in the new coordinates, gives a minimal value for the functional (11). After this, the smallness condition (10) will turn into

$$
f_{B_{R_{2}}^{+}(0)} e[u](x) d x<c_{*} \theta_{0}^{2}=\theta^{2}
$$

where the constant $c_{*}$ does not depend on $x^{0} \in \partial \Omega \cap \Omega_{0}$ but is determined by the parameters in the assumptions of Theorem 1.

In order to prove Theorem 3, we show that if $\theta_{0}$ and $R_{0}$ are sufficiently small (consequently, $\theta$ and $R_{2}$ in (12) ) are also small), then $u \in \mathcal{C}^{1, \beta}\left(\overline{B_{\tau R_{2}}^{+}}\right)$for some $\beta, \tau \in(0,1 / 2)$.

Under conditions $\left[\mathcal{A}_{3}\right]$, the expression $\widehat{a}|w|^{2}+\widehat{f} w$ in the integrand in (11) does not create additional complications when we study the regularity of the function $u$ at which the minimum in problem (11) is attained. So, in what follows we assume for simplicity that $\widehat{a}=\widehat{f}=0$ and write $A$ in place of $\widehat{A}$.

Thus, preserving the notation for the variables and the functions, we are going to show that the function $u$ at which the minimum of the functional

$$
F_{1}\left[w ; B_{R_{2}}^{+}\right]=\frac{1}{2} \int_{B_{R_{2}}^{+}(0)}\left(A(x, w) w_{x}, w_{x}\right) d x \mapsto \min _{W_{\mathcal{K}}^{+}}
$$

is attained, is smooth in $\overline{B_{\tau R_{2}}^{+}(0)}, \tau \in(0,1 / 2)$, provided (12) is true with $\theta_{2}$ and $R_{2}$ sufficiently small. The parameters $\beta$ and $\tau$ do not depend on $x^{0} \in \partial \Omega \cap \Omega_{0}$ and are determined by the data (11), (21), (5) of the problem.

Without loss of generality, we assume that

$$
a^{n n}(x) \equiv 1, \quad x \in B_{R_{2}}^{+} ;\left.\quad a_{n \tau}\right|_{\Gamma_{R_{2}}}=0, \quad \tau=1, \ldots, n-1
$$

(see, e.g., 39]). 
We shall prove that $u$ is smooth by the local penalty method. To construct the penalty, we consider the following scalar function: $\chi(s)=s$ for $s \leq \delta_{0}^{2} ; \chi(s)=-1 / 6\left(\frac{s^{2}}{\delta_{0}^{2}}-8 s+\delta_{0}^{2}\right)$ for $s \in\left(\delta_{0}^{2}, 4 \delta_{0}^{2}\right) ; \chi(s)=5 / 2 \delta_{0}^{2}$ for $s>4 \delta_{0}^{2}$. The function $\chi$ belongs to $C^{1,1}([0, \infty))$, and

$$
\chi^{\prime}(s) \geq 0, \quad\left|\chi^{\prime \prime}(s)\right| s \leq \frac{4}{3 \delta_{0}^{2}} \chi(s) .
$$

Here $\delta_{0}$ is the parameter taken from condition $\left[\mathbb{A}_{\mathcal{K}}\right]$.

For an arbitrary $\epsilon \in(0,1]$, consider the variational problem

$$
\begin{aligned}
F_{1}^{\epsilon}[v]= & \frac{1}{2} \int_{B_{R_{2}}^{+}}\left[\left(A(x, v) v_{x}, v_{x}\right)+|v-u|^{2}\right] d x \\
& +\frac{1}{2 \epsilon} \int_{B_{R_{2}}^{+}} \chi\left(d^{2}(v)\right) d x+\frac{1}{2 \epsilon} \int_{\Gamma_{R_{2}}} \chi\left(d^{2}(v)\right) d \Gamma \mapsto \min _{V},
\end{aligned}
$$

where

$$
V=\left\{v \in W_{2}^{1}\left(B_{R_{2}}^{+}\right): v-\left.u\right|_{S_{R_{2}}^{+}}=0\right\} .
$$

For every fixed $\epsilon>0$, the variational problem (16), (17) admits a solution $u^{\epsilon} \in V$. Since $u \in \mathcal{K}$ almost everywhere in $\Omega$ and on $\partial \Omega$, we see that $\chi\left(d^{2}(u)\right)=0$ a.e. in $\Omega$ and on $\partial \Omega$. Consequently,

$$
F_{1}^{\epsilon}\left[u^{\epsilon}\right] \leq F_{1}^{\epsilon}[u]=F_{1}[u] .
$$

It follows that

$$
\begin{gathered}
\left\|u_{x}^{\epsilon}\right\|_{2, B_{R_{2}}^{+}} \leq c\left\|u_{x}\right\|_{2, B_{R_{2}}^{+}} ; \quad\left\|u^{\epsilon}\right\|_{2, B_{R_{2}}^{+}} \leq c\|u\|_{W_{2}^{1}\left(B_{R_{2}}^{+}\right)} ; \\
\frac{1}{\epsilon} \int_{B_{R_{2}}^{+}} \chi\left(d^{2}\left(u^{\epsilon}\right)\right) d x \leq c\left\|u_{x}\right\|_{2, B_{R_{2}}^{+}}^{2} ; \quad \frac{1}{\epsilon} \int_{\Gamma_{R_{2}}} \chi\left(d^{2}\left(u^{\epsilon}\right)\right) d \Gamma \leq c\left\|u_{x}\right\|_{2, B_{R_{2}}^{+}}^{2} .
\end{gathered}
$$

By (19), there is a function $u^{0} \in W_{2}^{1}\left(B_{R_{2}}^{+}\right)$such that, for some sequence of values of $\epsilon \rightarrow 0$, the sequence $u^{\epsilon}$ converges weakly to $u_{0}$ in $W_{2}^{1}\left(B_{R_{2}}^{+}\right)$. Moreover, $u^{0} \in \mathcal{K}$ a.e. in $B_{R_{2}}^{+}$and on $\Gamma_{R_{2}}, u^{0}=u$ on $S_{R_{2}}^{+}$. Next,

$$
F_{1}\left[u^{\epsilon}\right] \leq F_{1}^{\epsilon}\left[u^{\epsilon}\right] \leq F_{1}[u]
$$

and

$$
F_{1}\left[u^{0}\right] \leq \liminf _{\epsilon} F_{1}\left[u^{\epsilon}\right] \leq \limsup _{\epsilon} F_{1}\left[u^{\epsilon}\right] \leq F_{1}[u] \leq F_{1}\left[u^{0}\right] .
$$

The last inequality is true because the function $u$ is minimal for the functional $F_{1}$. Thus, equality occurs throughout in the chain (20) of inequalities, whence we see that the following limit exists:

$$
\lim _{\epsilon} F_{1}\left[u^{\epsilon}\right]=F_{1}[u] .
$$

From (18) and (21) we deduce that

$$
\begin{aligned}
\lim _{\epsilon} \int_{B_{R_{2}}^{+}}\left|u^{\epsilon}-u\right|^{2} d x & =\int_{B_{R_{2}}^{+}}\left|u^{0}-u\right|^{2} d x=0, \\
\lim _{\epsilon} \frac{1}{\epsilon} \int_{B_{R_{2}}^{+}} \chi\left(d^{2}\left(u^{\epsilon}\right)\right) d x & =0, \quad \lim _{\epsilon} \frac{1}{\epsilon} \int_{\Gamma_{R_{2}}} \chi\left(d^{2}\left(u^{\epsilon}\right)\right) d \Gamma=0 .
\end{aligned}
$$

Thus, $u^{0}=u$ in $B_{R_{2}}^{+}$. Since the $u^{\epsilon}$ converge to $u$ weakly in $W_{2}^{1}\left(B_{R_{2}}^{+}\right)$, by (21) we see that $u^{\epsilon}$ tends to $u$ in the norm of $W_{2}^{1}\left(B_{R_{2}}^{+}\right)$. Therefore, we have proved the following assertion. 
Proposition 1. The solutions $u^{\epsilon}$ of the penalty problems (16), (17) tend to the solution $u$ under study for the problem (13) in the norm of $W_{2}^{1}\left(B_{R_{2}}^{+}\right)$for some sequence $\epsilon \rightarrow 0$, and formulas (22) hold true.

In what follows, we shall write

$$
\chi_{\epsilon}\left(d^{2}\left(u^{\epsilon}\right)\right)=\frac{1}{\epsilon} \chi\left(d^{2}\left(u^{\epsilon}\right)\right) .
$$

§3. Monotonicity INEQUALITy FOR THE NORMALIZED ENERGY INTEGRAL

We put

$$
\begin{aligned}
& \Phi^{\epsilon}\left(r, x^{0}\right)=f_{\omega_{r}\left(x^{0}\right)} e^{\epsilon}\left[u^{\epsilon}\right](x) d x+\frac{1}{2} f_{\gamma_{r}\left(x^{0}\right)} \chi_{\epsilon}\left(d^{2}\left(u^{\epsilon}\right)\right) d \gamma \\
& e^{\epsilon}\left[u^{\epsilon}\right](x)=\frac{1}{2}\left[\left(A\left(x, u^{\epsilon}\right) u_{x}^{\epsilon}, u_{x}^{\epsilon}\right)+\left|u^{\epsilon}-u\right|^{2}+\chi_{\epsilon}\left(d^{2}\left(u^{\epsilon}\right)\right)\right],
\end{aligned}
$$

where $\chi_{\epsilon}$ is defined by (23), $\omega_{r}\left(x^{0}\right)=B_{R_{2}}^{+}(0) \cap B_{r}\left(x^{0}\right), \gamma_{r}\left(x^{0}\right)=\Gamma_{R_{2}}(0) \cap B_{r}\left(x^{0}\right)$, $x^{0} \in B_{R_{2}} \cup \Gamma_{R_{2}}$, and $r<\operatorname{dist}\left(x^{0}, S_{R_{2}}^{+}\right)$.

Remark 2. Proposition 1 and condition (12) show that for some sequence of $\epsilon \rightarrow 0$, we have

$$
\Phi^{\epsilon}\left(R_{2}, 0\right)<\theta^{2}, \quad \epsilon \leq \epsilon_{*} .
$$

In the sequel we only consider this sequence of $\epsilon \rightarrow 0$.

Proposition 2. If (25) is fulfilled, there exist numbers $\tau_{1} \in(0,1 / 2)$ and $c_{1}>0$ such that

$$
\Phi^{\epsilon}\left(\rho, x^{0}\right) \leq c_{1}\left(\Phi^{\epsilon}\left(r, x^{0}\right)+\theta^{2}\right), \quad x^{0} \in \overline{B_{R_{3}}^{+}}, \quad \rho \leq r \leq R_{3}=\tau_{1} R_{2} .
$$

The constants $\tau_{1}$ and $c_{1}$ do not depend on $\epsilon \leq \epsilon_{*}$.

Proof. The arguments presented below are a modification of the method suggested in 38 for the proof of a monotonicity formula for harmonic mappings into the sphere.

Let $u^{\epsilon}$ be the solution of the variational problem (16), (17). Then this function is a critical point for the functional $F_{1}^{\epsilon}[\cdot]$ both relative to variation of dependent variables and relative to variation of independent variables. This means that we can fix a family of smooth homeomorphisms

$$
\psi_{\tau}(x)=x+\tau \xi(x)=x^{(\tau)}, \quad x \in B^{+}=B_{R_{2}}^{+}(0),
$$

such that $\psi_{\tau}: B^{+} \rightarrow B^{+}$for $0<\tau \ll 1$. Here $\xi=\left(\xi^{1}, \ldots, \xi^{n}\right), \xi \in \mathcal{C}^{(0,1)}\left(\overline{B^{+}}\right)$, $\operatorname{spt} \xi \subset B^{+} \cup \Gamma, \Gamma=\Gamma_{R_{2}}(0), \xi^{n} \geq 0$ in $B^{+},\left.\xi^{n}\right|_{\Gamma}=0$, and $\psi_{\tau}(\Gamma) \subset \Gamma$.

For $\epsilon$ fixed, we calculate the expression

$$
I_{\epsilon}(\tau)=\tau^{-1}\left(F_{1}^{\epsilon}\left[u^{\epsilon}\left(x^{(\tau)}\right)\right]-F_{1}^{\epsilon}\left[u^{\epsilon}(x)\right]\right) .
$$

The quantity $I_{\epsilon}(\tau)$ tends to a finite limit as $\tau \rightarrow+0$, and

$$
\lim _{\tau \rightarrow+0} I_{\epsilon}=\left.\frac{d F_{1}^{\epsilon}\left[u^{\epsilon}\left(x^{(\tau)}\right]\right.}{d \tau}\right|_{\tau=+0} \geq 0
$$

because $u^{\epsilon}$ is minimal. 
Inequality (27) can be rewritten as the following integral inequality for the function $u^{\epsilon}$ :

$$
\begin{aligned}
& -\int_{B^{+}} e^{\epsilon}\left[u^{\epsilon}\right] \operatorname{div} \xi d x+\int_{B^{+}} A_{k l}^{\alpha \beta}\left(u^{\epsilon}\right)_{x_{\beta}}^{k}\left(u^{\epsilon}\right)_{x_{\gamma}}^{l} \xi_{x_{\alpha}}^{\gamma} d x \\
& \quad-\frac{1}{2} \int_{B^{+}}\left(A_{k l}^{\alpha \beta}\right)_{x_{\gamma}}^{\prime}\left(u^{\epsilon}\right)_{x_{\beta}}^{l}\left(u^{\epsilon}\right)_{x_{\alpha}}^{k} \xi^{\gamma} d x+\int_{B^{+}}\left(u^{\epsilon}-u\right) u_{x_{\gamma}} \xi^{\gamma} d x \\
& \quad-\frac{1}{2} \int_{\Gamma} \chi_{\epsilon}\left(d^{2}\left(u^{\epsilon}\right)\right) \operatorname{div}^{\prime} \xi d \gamma \geq 0,
\end{aligned}
$$

where $\xi \in \mathcal{C}^{0,1}\left(\overline{B^{+}}\right), \xi^{n} \geq 0$ in $B^{+},\left.\xi^{n}\right|_{\Gamma}=0,\left.\xi\right|_{S^{+}}=0$, and $\operatorname{div}^{\prime} \xi=\sum_{\alpha \leq n-1} \xi_{x_{\alpha}}^{\alpha}$. The expression $e^{\epsilon}\left[u^{\epsilon}\right]$ was defined in (24), and $u$ is the solution under study of problem (13).

Now we fix $x^{0} \in \Gamma_{R / 2}(0)$ and $R \leq R_{2} / 2$. Then $\omega_{R}\left(x^{0}\right)=B^{+} \cap B_{R}\left(x^{0}\right)=B_{R}^{+}\left(x^{0}\right)$, $\gamma_{R}\left(x^{0}\right)=\Gamma \cap B_{R}\left(x^{0}\right)$. Suppose that spt $\xi \subset \omega_{R}\left(x^{0}\right) \cup \gamma_{R}\left(x^{0}\right)$ in (28). We can write

$$
e\left[u^{\epsilon}\right] \equiv \frac{1}{2} A_{k l}^{\alpha \beta}\left(x, u^{\epsilon}\right)\left(u^{\epsilon}\right)_{x_{\beta}}^{l}\left(u^{\epsilon}\right)_{x_{\alpha}}^{k}=e_{0}\left[u^{\epsilon}\right]+\frac{1}{2} \Delta a^{\alpha \beta} b_{k l}\left(x, u^{\epsilon}\right)\left(u^{\epsilon}\right)_{x_{\beta}}^{l}\left(u^{\epsilon}\right)_{x_{\alpha}}^{k},
$$

where

$$
\begin{aligned}
e_{0}\left[u^{\epsilon}\right] & =\frac{1}{2} a^{\alpha \beta}\left(x^{0}\right) b_{k l}\left(x, u^{\epsilon}\right)\left(u^{\epsilon}\right)_{x_{\beta}}^{l}\left(u^{\epsilon}\right)_{x_{\alpha}}^{k}, \\
\Delta a & =a(x)-a\left(x^{0}\right), \quad|\Delta a| \leq c\left|x-x^{0}\right| .
\end{aligned}
$$

By an orthogonal transformation of the coordinates $x$, we can reduce the matrix $a\left(x^{0}\right)$ in the above integral inequality to a diagonal form, after which it can be transformed to the unit matrix. Similar transformations were presented in detail in the author's paper 15] for the problem with penalty $\chi_{\epsilon}$ in $\omega_{R}\left(x^{0}\right)$, and in the author's paper [30] for the problem with penalty $\chi_{\epsilon}$ on $\gamma\left(x^{0}\right)$. Here we only give the further arguments for the simplest case where $a(x)=$ id (i.e., the unit matrix), and $b=b(u)$. Then (28) takes the form

$$
\begin{aligned}
& -\int_{\omega_{r}\left(x^{0}\right)} e^{\epsilon}\left[u^{\epsilon}\right] \operatorname{div} \xi d x+\int_{\omega_{R}\left(x^{0}\right)} b_{k l}\left(u^{\epsilon}\right)_{x_{\alpha}}^{l}\left(u^{\epsilon}\right)_{x_{\gamma}}^{k} \xi_{x_{\alpha}}^{\gamma} d x \\
& +\int_{\omega_{R}\left(x^{0}\right)}\left(u^{\epsilon}-u\right) u_{x_{\gamma}} \xi^{\gamma} d x-\frac{1}{2} \int_{\gamma\left(x^{0}\right)} \chi_{\epsilon} \operatorname{div}^{\prime} \xi d \gamma \geq 0
\end{aligned}
$$

where $\xi$ is as in (28).

Fixing two numbers $r$ and $r+h \leq R$ arbitrarily, we define a function $\eta$ by $\eta(s)=1$ for $s \leq r$ and $\eta(s)=\frac{r-s}{h}+1$ for $s \in(r, r+h]$, and put $\xi=\left(x-x^{0}\right) \eta\left(\left|x-x^{0}\right|\right)$ in (29). (Recall that $x^{0} \in \Gamma_{R_{2}}$, whence $x_{n}^{0}=0$ and $\left.\xi^{n}\right|_{\Gamma_{R_{2}}}=0$.) After some calculations, we let $h$ tend to zero, and we arrive at the relation

$$
\begin{aligned}
(2-n) & \int_{B_{r}^{+}\left(x^{0}\right)} e^{\epsilon}\left[u^{\epsilon}\right] d x+r \int_{S_{r}^{+}\left(x^{0}\right)} e^{\epsilon}\left[u^{\epsilon}\right] d s \\
& -\frac{1}{r} \int_{S_{r}^{+}\left(x^{0}\right)} b_{k l}\left(\left(u^{\epsilon}\right)_{x}^{l},\left(x-x^{0}\right)\right)\left(\left(u^{\epsilon}\right)_{x}^{k},\left(x-x^{0}\right)\right) d s \\
& -\int_{B_{r}^{+}\left(x^{0}\right)}\left[\chi_{\epsilon}\left(d^{2}\left(u^{\epsilon}\right)\right)+\left|u^{\epsilon}-u\right|^{2}\right] d x+\int_{B_{r}^{+}\left(x^{0}\right)}\left(\left(u^{\epsilon}-u\right),\left(u_{x},\left(x-x^{0}\right)\right)\right) d x \\
& -\frac{(n-1)}{2} \int_{\gamma_{r}\left(x^{0}\right)} \chi_{\epsilon} d \gamma+\frac{r}{2} \int_{\partial \gamma_{r}\left(x^{0}\right)} \chi_{\epsilon} d(\partial \gamma) \geq 0 .
\end{aligned}
$$


Dividing this by $r^{n-1}$, we readily obtain the inequality

$$
\begin{aligned}
& \left(-\frac{(n-2)}{r^{n-1}} \int_{B_{r}^{+}} e^{\epsilon} d x+\frac{1}{r^{n-2}} \int_{S_{r}^{+}} e^{\epsilon} d s\right) \\
& \quad+\left(-\frac{(n-2)}{r^{n-1}} \int_{\gamma_{r}} \frac{1}{2} \chi_{\epsilon} d \gamma+\frac{1}{r^{n-2}} \int_{\partial \gamma} \frac{1}{2} \chi_{\epsilon} d(\partial \gamma)\right) \\
& \geq \frac{1}{r^{n}} \int_{S_{r}^{+}} b_{k l}\left(\left(u^{\epsilon}\right)_{x}^{l}, x-x^{0}\right)\left(\left(u^{\epsilon}\right)_{x}^{k}, x-x^{0}\right) d s \\
& \quad+\frac{1}{r^{n-1}} \int_{B_{r}^{+}}\left(\chi_{\epsilon}+\left|u^{\epsilon}-u\right|^{2}\right) d x-\frac{1}{r^{n-1}} \int_{B_{r}^{+}}\left(u^{\epsilon}-u,\left(u_{x}, x-x^{0}\right)\right) d x .
\end{aligned}
$$

Now, the left-hand side of (30) is $\left(\Phi^{\epsilon}\left(r, x^{0}\right)\right)_{r}^{\prime}$, and the first integral on the right is nonnegative (the latter is because of a split structure of $A$ ). So, we see that

$$
\begin{aligned}
\left(\Phi^{\epsilon}\left(r, x^{0}\right)\right)_{r}^{\prime} & \geq \frac{1}{r^{n-1}} \int_{B_{r}^{+}}\left|u^{\epsilon}-u\right|^{2} d x-f_{B_{r}^{+}}\left|u^{\epsilon}-u\right|\left|u_{x}\right| d x \\
& \geq \frac{1}{2 r^{n-1}} \int_{B_{r}^{+}}\left|u^{\epsilon}-u\right|^{2} d x-\frac{r}{2} f_{B_{r}^{+}}\left|u_{x}\right|^{2} d x .
\end{aligned}
$$

Integrating over the interval $(\rho, r)$, we obtain

$$
\begin{aligned}
\Phi^{\epsilon}\left(r, x^{0}\right)-\Phi^{\epsilon}\left(\rho, x^{0}\right) & \geq \int_{\rho}^{r}\left(\frac{1}{t^{n-1}} \int_{B_{t}^{+}}\left|u^{\epsilon}-u\right|^{2} d x-f_{B_{t}^{+}}\left|u^{\epsilon}-u\right|\left|u_{x}\right| d x\right) d t \\
& \geq-\frac{1}{2} \int_{\rho}^{r} t\left(f_{B_{t}^{+}}\left|u_{x}\right|^{2} d x\right) d t,
\end{aligned}
$$

where $0<\rho<r \leq R$. To deduce (26) from (31), we need some additional information about the limit function $u(x)$. For this, we put $r=R=R_{2} / 2$ in the first inequality in (31), obtaining

$$
\Phi^{\epsilon}\left(\rho, x^{0}\right) \leq \Phi^{\epsilon}\left(\frac{R_{2}}{2}, x^{0}\right)+\int_{\rho}^{R_{2} / 2}\left(f_{B_{t}^{+}}\left|u^{\epsilon}-u\right|\left|u_{x}\right| d x\right) d t .
$$

Since $\Phi^{\epsilon}\left(R_{2} / 2, x^{0}\right) \leq 2^{n-2} \Phi^{\epsilon}\left(R_{2}, 0\right) \leq 2^{n-2} \theta^{2}$, letting $\epsilon$ tend to 0 , from the last inequality and (22) we derive that

$$
f_{B_{\rho}^{+}\left(x^{0}\right)}\left|u_{x}\right|^{2} d x \leq c \theta^{2}, \quad x^{0} \in \Gamma_{R_{2} / 2}(0), \quad \rho \leq R_{2} / 2 .
$$

Clearly, for every $x^{0} \in B_{R_{2} / 4}^{+}(0)$ and every $r_{0} \leq d_{0}=\operatorname{dist}\left(x^{0}, \Gamma_{R_{2}}\right)$, we can use (28) with $\xi \in \mathcal{C}^{0,1}\left(\overline{B_{r_{0}}\left(x^{0}\right)}\right)$ to obtain an inequality of the form (31) for $0<\rho<r \leq r_{0}$. The limit passage as $\epsilon \rightarrow 0$ in the resulting relation shows that

$$
f_{B_{\rho}\left(x^{0}\right)}\left|u_{x}\right|^{2} d x \leq c \theta^{2}, \quad \rho \leq d_{0}, \quad x^{0} \in B_{R_{2} / 4}^{+}(0) .
$$

The usual procedure of "sewing" the boundary estimate (32) and the inner estimate (33) guarantees that

$$
\sup _{x^{0} \in B_{R_{2} / 4}^{+}, \rho \leq R_{2} / 4} f_{\omega_{\rho}\left(x^{0}\right)}\left|u_{x}\right|^{2} d x \leq c \theta^{2}
$$

for the limit function $u(x)$, i.e., $u_{x} \in L^{2, n-2}\left(B_{R_{2} / 4}^{+}(0)\right)$; moreover, the seminorm [.] of the Campanato space $\mathcal{L}^{2, n}\left(B_{R_{2} / 4}^{+}\right)$is estimated as follows:

$$
[u]_{\mathcal{L}^{(2, n)}\left(B_{R_{2} / 4}^{+}\right)}^{2} \leq c \theta^{2} .
$$


Estimates (34) and (35) for the limit function are useful in the current proof, but they will also be useful in the sequel.

Returning to (31) with $x^{0} \in \Gamma_{R_{2} / 2}$, by the invocation of (32) we conclude that

$$
\Phi^{\epsilon}\left(\rho, x^{0}\right) \leq \Phi^{\epsilon}\left(r, x^{0}\right)+c \theta^{2}\left(r^{2}-\rho^{2}\right),
$$

$\rho<r \leq R_{2} / 2, x^{0} \in \Gamma_{R_{2} / 2}$. Similarly, by using (33) for the limit function $u$, we obtain an inequality such as (36) for interior points $x^{0} \in B_{R_{2} / 4}(0)$ and $r \leq \operatorname{dist}\left(x^{0}, \Gamma_{R_{2} / 4}\right)$. This implies the general result. It should be noted that, in the case where the matrix $a_{\alpha \beta}$ is of general form, an inequality such as (30) and all the subsequent estimates will involve junior terms immaterial for the proof; see [30].

Remark 3. As was noted in Remark 2, condition (12) implies estimate (25). Taking (26) into account, we conclude that (12) guarantees the existence of a constant $c_{2}>0$ (which depends on the problem data) with

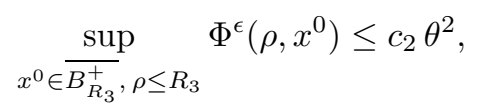

where $R_{3}=\tau_{1} R_{2}$ was fixed in Proposition 2 .

Remark 4. Estimate (35) shows that the limit function $u(x)$ belongs to the Campanato space $\mathcal{L}^{2, n}\left(B_{R_{3}}^{+}\right), R_{3}<R_{2}$; consequently, $u \in L_{m}\left(B_{R_{3}}^{+}\right)$for all $m<\infty$ and, moreover,

$$
\|u\|_{m, B_{R_{3}}^{+}}^{m} \leq c(m) \theta^{m} R_{3}^{n}+c R_{3}^{n(1-m / 2)}\|u\|_{2, B_{R_{3}}^{+}}^{m} \equiv \widehat{c}\left(m, n, R_{3}^{-1},\|u\|_{2, B_{R_{3}}^{+}}\right) .
$$

\section{§4. The SMOOThNeSS OF $u^{\epsilon}$ NEAR THE ORIGIN}

Generally speaking, the vector-valued functions $u^{\epsilon}$ at which the functional $F_{1}^{\epsilon}[\cdot]$ of problem (16), (17) attains its minimum may have a singular set $\Sigma_{\epsilon} \subset B_{R_{2}}^{+} \cup \Gamma_{R_{2}}$. Nevertheless, we shall show that, in our case, estimate (37) with $\theta$ sufficiently small guarantees that $u^{\epsilon} \in \mathcal{C}^{1, \gamma}\left(\overline{B_{R_{4}}^{+}}\right), R_{4}<R_{3}$, for every $\gamma \in(0,1)$ (however, the corresponding norm may grow as $\epsilon \rightarrow 0$ ). It is important that $R_{4}$ does not depend on $\epsilon$. This preliminary information about the smoothness of $u^{\epsilon}$ will be required in the next section to estimate $\left\|u_{x}^{\epsilon}\right\|_{\infty, B_{R}^{+}}$uniformly in $\epsilon$ for some $R<R_{4}$.

Proposition 3. There exist $\theta>0, R_{2}>0$ such that, whenever a solution $u^{\epsilon}, \epsilon \leq \epsilon_{*}$, of problem (16), (17) satisfies (25), we have $u^{\epsilon} \in \mathcal{C}^{1, \gamma}\left(\overline{B_{R_{4}}^{+}}\right) \cap W_{2}^{2}\left(B_{R_{4}}^{+}\right)$for every $\gamma \in(0,1)$ and $R_{4}=\tau_{2} R_{2}$; moreover, the parameter $\tau_{2}<1$ does not depend on $\epsilon$. Next,

$$
\left\|u^{\epsilon}\right\|_{\mathcal{C}^{1, \gamma}\left(\overline{B_{R_{4}}^{+}}\right)}+\left\|u_{x x}^{\epsilon}\right\|_{2, B_{R_{4}}^{+}} \leq K\left(\epsilon^{-1}\right),
$$

where $K\left(\epsilon^{-1}\right)$ may tend to infinity as $\epsilon \rightarrow 0$.

Proof. First, we observe that, under condition (25), estimates (26) and (37) are valid in $B_{R_{3}}^{+}$, where $R_{3}^{+}=\tau_{1} R_{2}, \tau_{1} \leq 1 / 2$. In order to show that $u^{\epsilon}$ is Hölder continuous in some closed half-ball $\overline{B_{R}^{+}(0)}, R<R_{3}, \epsilon \leq \epsilon_{*}$, we apply the "direct" method of checking smoothness for solutions (minimizers rather than arbitrary extremal elements) of variational problems; see [31, Chapter 6]). At the first step, we estimate $\left|u_{x}^{\epsilon}\right|$ in $L_{p}$ on $B_{R}^{+}$for some $p>2$ and some $R<R_{3}$, say, $R=R_{3} / 2$ :

$$
\left\|u_{x}^{\epsilon}\right\|_{p, B_{R_{3} / 2}^{+}} \leq K_{1}\left(\epsilon^{-1}\right), \quad \epsilon \leq \epsilon_{*}
$$

The exponent $p>2$ only depends on the ellipticity constants for the matrix $A$ and on the dimension $n$. The volume and surface penalty integrals will appear in the estimate as supplementary terms with coefficient $\frac{1}{\epsilon}$. 
Next, we freeze the arguments of the matrix $A(x, u)$ and show that

$$
\left\|u^{\epsilon}\right\|_{\mathcal{C}^{\alpha}\left(\overline{B_{R_{3} / 2}^{+}}\right)} \leq K_{2}\left(\epsilon^{-1}\right), \quad \alpha \in(0,1),
$$

provided $\theta$ and $R_{2}$ are sufficiently small (they will depend on $A$ but not on $\epsilon$ ). After that, we regard $u^{\epsilon}$ as an extremal element for the functional $F_{1}^{\epsilon}$ that is a weak solution of the following boundary-value problem:

$$
\begin{gathered}
L_{\epsilon}^{(k)}\left[u^{\epsilon}\right] \equiv-\left(A_{k l}^{\alpha \beta}\left(x, u^{\epsilon}\right)\left(u^{\epsilon}\right)_{x_{\beta}}^{l}\right)_{x_{\alpha}}+\frac{1}{2}\left(A_{m l}^{\alpha \beta}\right)_{u^{k}}^{\prime}\left(u^{\epsilon}\right)_{x_{\beta}}^{l}\left(u^{\epsilon}\right)_{x_{\alpha}}^{m} \\
+\left[\left(u^{\epsilon}\right)^{k}-u^{k}\right]+\frac{1}{2} \frac{d \chi_{\epsilon}\left(d^{2}\left(u^{\epsilon}\right)\right)}{d u^{k}}=0, \quad x \in B_{R}^{+}(0), \\
A_{k l}^{n \beta}\left(x, u^{\epsilon}\right)\left(u^{\epsilon}\right)_{x_{\beta}(14)}^{l} b_{k l}\left(x, u^{\epsilon}\right)\left(u^{\epsilon}\right)_{x_{n}}^{l}=\frac{1}{2} \frac{d \chi_{\epsilon}}{d u^{k}}, \quad x \in \Gamma_{R}, \quad R=R_{3} / 2, \quad k \leq N .
\end{gathered}
$$

The function $u^{\epsilon} \in W_{2}^{1}\left(B_{R}^{+}\right) \cap \mathcal{C}^{\alpha}\left(\overline{B_{R}^{+}}\right)$satisfies the identity

$$
\begin{aligned}
& \int_{B_{R}^{+}}\left[A_{k l}^{\alpha \beta}\left(x, u^{\epsilon}\right)\left(u^{\epsilon}\right)_{x_{\beta}}^{l} h_{x_{\alpha}}^{k}+\right. \frac{1}{2}\left(A_{m l}^{\alpha \beta}\right)_{u^{k}}^{\prime}\left(u^{\epsilon}\right)_{x_{\beta}}^{l}\left(u^{\epsilon}\right)_{x_{\alpha}}^{m} h^{k}+\left(\left(u^{\epsilon}\right)^{k}-u^{k}\right) h^{k} \\
&+\left.\frac{1}{2} \frac{d \chi_{\epsilon}}{d u^{k}} h^{k}\right] d x+\int_{\Gamma_{R}(0)} \frac{1}{2} \frac{d \chi_{\epsilon}}{d u^{k}} h^{k} d \Gamma=0 \\
& h \in W_{2}^{1}\left(B_{R}^{+}\right) \cap L_{\infty}\left(B_{R}^{+}\right),\left.\quad h\right|_{S_{R}^{+}}=0 .
\end{aligned}
$$

A local estimate for the Hölder norm of the gradient for the solution of a strongly nonlinear elliptic system was obtained in 33; an estimate near the boundary for the gradient under the Dirichlet condition and a nonlinear boundary condition of Neumann type were established by the author in 34 .

So, by [34], the solutions $u^{\epsilon}$ of problem (41) belong to $\mathcal{C}^{1, \gamma}\left(\overline{B_{R / 2}^{+}}\right)$for every $\gamma \in(0,1)$, and $\left\|u^{\epsilon}\right\|_{\mathcal{C}^{1, \gamma}\left(\overline{B_{R}^{+}}\right)} \leq K_{3}\left(\epsilon^{-1}, \gamma\right), R \leq \frac{R}{16}$.

The last estimate allows us to view problem (40) as a linear problem with the Neumann condition on $\Gamma_{R}$ and to conclude that, at least, $\left\|u_{x x}^{\epsilon}\right\|_{2, B_{R}^{+}} \leq k_{4}\left(\epsilon^{-1}\right)$. Thus, Proposition 3 is proved with $R_{4}=\frac{R_{3}}{32}=\tau_{2} R_{2}$.

The information obtained above makes it possible to pass to estimating $\left\|u_{x}^{\epsilon}\right\|_{\infty, B_{R_{5}}^{+}}$ uniformly in $\epsilon \leq \epsilon_{*}$ in some half-ball $B_{R_{5}}^{+}$, where $R_{5}<R_{4}$ does not depend on $\epsilon \leq \epsilon_{*}$.

$$
\text { §5. An estimate OF }\left\|u_{x}^{\epsilon}\right\|_{\infty, B_{R}^{+}} \text {UNIFORM in } \epsilon \leq \epsilon_{*}
$$

In this section, we show that the function

$$
p\left[u^{\epsilon}\right](x)=\frac{1}{2} \sum_{\alpha \leq n} b_{k l}\left(x, u^{\epsilon}\right)\left(u^{\epsilon}\right)_{x_{\alpha}}^{l}\left(u^{\epsilon}\right)_{x_{\alpha}}^{k}+\frac{1}{2} \chi_{\epsilon}\left(d^{2}\left(u^{\epsilon}\right)\right)
$$

is bounded in $B_{R_{5}}^{+}$with some $R_{5}<R_{1}$, uniformly in $\epsilon \leq \epsilon_{*}$, provided that (37) is fulfilled with $\theta$ sufficiently small.

To do this, it is useful to observe that the integrals

$$
f_{\omega_{r}\left(x^{0}\right)}\left|u_{x}^{\epsilon}\right|^{2} d x, \quad \omega_{r}\left(x^{0}\right)=B_{r}^{+}\left(x^{0}\right) \cap B_{R_{4}}^{+}, \quad x^{0} \in \overline{B_{R_{4}}^{+}}, \quad r<R_{4},
$$

are invariant under the transformation $y=\lambda\left(x-x^{0}\right), \lambda=$ const $>0$. This makes it possible to fix a conjectural maximum point $x^{0}$ for the function $p\left[u^{\epsilon}\right](x)$ and, in some neighborhood $\omega_{r}\left(x^{0}\right)$, consider a transformation $y=\lambda\left(x-x^{0}\right)$ such that the function 
$\widetilde{p}\left[v^{\epsilon}\right]=\frac{p\left[u^{\epsilon}\right]}{\lambda^{2}}, v^{\epsilon}(y)=u^{\epsilon}\left(x^{0}+y / \lambda\right)$, is bounded on the set $\widehat{\omega}_{\lambda r}(0)=y\left(\omega_{r}\left(x^{0}\right)\right)$ uniformly in $\epsilon \leq \epsilon_{*}$. Condition (37) and the boundedness of the functions $\widetilde{p}\left[v^{\epsilon}\right]$ imply that

$$
\|\widetilde{p}\|_{s, \hat{\omega}_{\lambda r}(0)}^{s} \leq c(s) \theta^{2}, \quad s \geq 1 .
$$

Also, we observe that, by using the split structure (5) of $A$, we can show that the scalar functions

$$
p_{1}\left[u^{\epsilon}\right](x)=\sum_{\tau \leq n-1} b_{k l}\left(x, u^{\epsilon}\right)\left(u^{\epsilon}\right)_{x_{\tau}}^{l}\left(u^{\epsilon}\right)_{x_{\tau}}^{k}+\chi_{\epsilon}\left(d^{2}\left(u^{\epsilon}\right)\right)
$$

and

$$
p_{2}\left[u^{\epsilon}\right](x)=b_{k l}\left(x, u^{\epsilon}\right)\left(u^{\epsilon}\right)_{x_{n}}^{l}\left(u^{\epsilon}\right)_{x_{n}}^{k}+\chi_{\epsilon}\left(d^{2}\left(u^{\epsilon}\right)\right)
$$

are subsolutions of the elliptic equation with the operator $L w=-\left(a^{\alpha \beta}(x) w_{x_{\beta}}\right)_{x_{\alpha}}$ and with supplementary terms involving $\left|u_{x}^{\epsilon}\right|^{4}$. After passage to the coordinates $y$ indicated above, the functions $\widetilde{p}_{i}\left[v^{\epsilon}\right]=\frac{p_{i}\left[u^{\epsilon}\right]}{\lambda^{2}}, i=1,2$, become bounded subsolutions of elliptic equations with supplementary terms of small $L_{s}$-norm, $s \geq 1$.

The facts explained above allow us to prove the following statement.

Proposition 4. There exist constants $\theta>0$ and $R_{2}>0$ such that, if (25) with these constants is fulfilled, then

$$
\begin{gathered}
\frac{\max }{B_{R_{5}}^{+}} p^{\epsilon}\left[u^{\epsilon}\right](x) \leq c_{3}\left(R_{5}^{-1}, \theta^{-1}\right), \\
\left\|u_{x x}^{\epsilon}\right\|_{2, B_{R_{5}}^{+}}+\left\|\frac{d \chi_{\epsilon}}{d u}\right\|_{2, B_{R_{5}}^{+}} \leq c_{4}\left(R_{5}^{-1}, \theta^{-1}\right), \quad \epsilon \leq \epsilon_{*},
\end{gathered}
$$

where $R_{5}=\tau_{3} R_{2}$ with some $\tau_{3} \in(0,1 / 2)$.

Proof. It should be noted that we shall use a modification of the method employed in 35 . for the study of solutions of penalty problems required for the investigation of harmonic mappings.

Let $\theta$ and $R_{2}$ be fixed in accordance with Proposition 3. Then $u^{\epsilon}$ is a smooth function in some half-ball $B_{R_{4}}^{+}, \epsilon \leq \epsilon_{*}$. We note that (25) implies (37).

For an arbitrary $R \leq R_{4}$, consider the quantity

$$
\max _{0 \leq \sigma \leq R}\left\{(R-\sigma)^{2} \frac{\max }{B_{\sigma}^{+}(0)} p^{\epsilon}\left[u^{\epsilon}\right](x)\right\}=\left(R-\sigma_{0}\right)^{2} \frac{\max }{B_{\sigma_{0}}^{+}} p^{\epsilon}\left[u^{\epsilon}\right],
$$

where $p^{\epsilon}\left[u^{\epsilon}\right]$ is defined by (42).

To simplify the arguments, we even omit the term involving $u^{\epsilon}-u$ in (41). By estimates (35) and (38) for the limit function $u$, this term does not bring about essential changes in the proof.

We fix $\sigma_{0} \in[0, R)$ by (44). Let $x^{0}$ be a point in $\overline{B_{\sigma_{0}}^{+}(0)}$ at which the function $p^{\epsilon}\left[u^{\epsilon}\right](x)$ attains its maximum. Put

$$
\begin{gathered}
e_{0}=p^{\epsilon}\left[u^{\epsilon}\right]\left(x^{0}\right)=\frac{\max }{B_{\sigma_{0}}^{+}} p^{\epsilon}\left[u^{\epsilon}\right](x), \quad \rho_{0}=\frac{R-\sigma_{0}}{2}, \\
\omega_{\rho_{0}}\left(x^{0}\right)=B_{R}^{+}(0) \cap B_{\rho_{0}}\left(x^{0}\right), \quad \gamma_{\rho_{0}}\left(x^{0}\right)=\Gamma_{R}(0) \cap B_{\rho_{0}}\left(x^{0}\right) .
\end{gathered}
$$

Changing the coordinates by the rule $y=\sqrt{e_{0}}\left(x-x^{0}\right)$, we set

$$
v^{\epsilon}(y)=u^{\epsilon}\left(x^{0}+\frac{y}{\sqrt{e_{0}}}\right), \quad r_{0}=\rho_{0} \sqrt{e_{0}} .
$$

Next, we denote

$$
y\left(\omega_{\rho_{0}}\left(x^{0}\right)\right)=\widehat{\omega}_{r_{0}}(0), \quad y\left(\gamma_{\rho_{0}}\left(x^{0}\right)\right)=\widehat{\gamma}_{r_{0}}(0) .
$$


By (41) (we omit the summand involving $\left(u^{\epsilon}-u\right)$ in it), we see that $v=v^{\epsilon}$ obeys the relation

$$
\begin{gathered}
\int_{\hat{\omega}_{r_{0}}(0)}\left[\widehat{a}^{\alpha \beta}(y) \widehat{b}_{k l}(y, v) v_{y_{\beta}}^{l} h_{y_{\alpha}}^{k}+\frac{1}{2} \widehat{a}^{\alpha \beta}\left(\hat{b}_{m l}\right)_{v^{k}}^{\prime} v_{y_{\beta}}^{l} v_{y_{\alpha}}^{m} h^{k}+\frac{1}{2} \frac{d \tilde{\chi}_{\epsilon}\left(d^{2}(v)\right)}{d v^{k}} h^{k}\right] d y \\
\quad+\int_{\hat{\gamma}_{r_{0}}(0)} \frac{1}{2} \frac{\hat{\chi}_{\epsilon}\left(d^{2}(v)\right)}{d v^{k}} h^{k} d \gamma=0, \\
h \in \mathcal{C}^{1}\left(\widehat{\omega}_{r_{0}}(0)\right),\left.\quad h\right|_{\partial^{\prime} \omega_{r_{0}}}=0, \quad \partial^{\prime} \omega_{r_{0}}(0)=\omega_{r_{0}}(0) \backslash \hat{\gamma}_{r_{0}}(0) .
\end{gathered}
$$

Here $\widehat{a}$ and $\hat{b}$ denote the functions $a$ and $b$ in the new coordinates, and the transformed penalty functions look like this:

$$
\tilde{\chi}_{\epsilon}(\cdot)=\frac{\chi_{\epsilon}(\cdot)}{e_{0}}, \quad \hat{\chi}_{\epsilon}(\cdot)=\frac{\chi_{\epsilon}(\cdot)}{\sqrt{e_{0}}} .
$$

Note that

$$
\sup _{\omega_{\rho_{0}}\left(x^{0}\right)} p^{\epsilon}\left[u^{\epsilon}\right] \leq \sup _{B_{\rho_{0}+\sigma_{0}}^{+}(0)} p^{\epsilon}\left[u^{\epsilon}\right] \underset{(44)}{\leq} 4 e_{0}
$$

consequently, for $\widehat{p}^{\epsilon}\left[v^{\epsilon}\right]=\frac{p^{\epsilon}\left[u^{\epsilon}\right]}{e_{0}}$ we have

$$
\hat{p}\left[v^{\epsilon}\right](0)=1, \quad \sup _{\hat{\omega}_{r_{0}}(0)} \hat{p}^{\epsilon}\left[v^{\epsilon}\right](y) \leq 4 .
$$

If $e_{0} \leq \frac{1}{\theta^{2}}$ (throughout, we assume that $\theta \leq 1$ ), formula (44) with $\sigma=R / 2$ implies that

$$
\sup _{B_{R / 2}^{+}(0)} p^{\epsilon}\left[u^{\epsilon}\right] \leq 4 e_{0} \leq 4 / \theta^{2}
$$

which yields the first estimate in (43) with $R_{5}=R_{4} / 2$ if we take $R=R_{4}$.

Now we analyze the opposite situation: $e_{0}>1 / \theta^{2}$, i.e.,

$$
\frac{1}{e_{0}}<\theta^{2}
$$

If, moreover,

$$
r_{0} \leq 2
$$

where $r_{0}$ is defined by (46), then, putting $\sigma=R / 2$ in (44), we see that

$$
(R / 2)^{2} \sup _{B_{R / 2}^{+}(0)} p^{\epsilon}\left[u^{\epsilon}\right] \leq\left(R-\sigma_{0}\right)^{2} e_{0}=4 \rho_{0}^{2} e_{0}=4 r_{0}^{2} \leq 16,
$$

yielding the first estimate in (43) with $R=R_{4}$.

Thus, in order to prove the first estimate in (43), it remains to consider the case where

$$
r_{0}>2
$$

and (50) is true.

We show that this case is impossible if $\theta$ is sufficiently small. We introduce the scalar functions

$$
H[v]=\frac{1}{2} \sum_{\tau \leq n-1} \widehat{b}_{k l}(y, v) v_{y_{\tau}}^{l} v_{y_{\tau}}^{k}, \quad Q[v]=\frac{1}{2} \widehat{b}_{k l}(y, v) v_{y_{n}}^{l} v_{y_{n}}^{k}, \quad v=v^{\epsilon}(y) .
$$

By (42) and (48), we have

$$
\hat{p}^{\epsilon}\left[v^{\epsilon}\right]=\frac{p^{\epsilon}\left[u^{\epsilon}\right]}{e_{0}}=H\left[v^{\epsilon}\right]+Q\left[v^{\epsilon}\right]+\frac{1}{2} \tilde{\chi}_{\epsilon}\left(d^{2}\left(v^{\epsilon}\right)\right) .
$$

We are going to show that under conditions (50), (52) we have

$$
\tilde{\chi}_{\epsilon}\left(d^{2}\left(v^{\epsilon}(0)\right)\right)+H\left[v^{\epsilon}\right](0) \leq \lambda_{1}(\theta), \quad Q\left[v^{\epsilon}\right](0) \leq \lambda_{2}(\theta),
$$


where $\lambda_{i}(\theta) \rightarrow 0, i=1,2$, as $\theta \rightarrow 0$. By (54) and (49), we obtain

$$
1=\widehat{p}^{\epsilon}\left[v^{\epsilon}\right](0) \leq \lambda_{1}(\theta)+\lambda_{2}(\theta) \rightarrow 0, \quad \theta \rightarrow 0 .
$$

Thus, if $\theta$ is sufficiently small, we arrive at a contradiction, so that (52) cannot be true under condition (50). As has already been mentioned, this guarantees the first estimate in (43).

To prove (54), first we invoke (47), putting $h=\left(v_{y_{\tau}} \phi\right)_{y_{\tau}}, \tau \leq n-1, \phi \in \mathcal{C}^{1}\left(\widehat{\omega}_{2} \cup \widehat{\gamma}_{2}\right)$, $\operatorname{spt} \phi \subset \widehat{\omega}_{2} \cup \hat{\gamma}_{2}, \phi \geq 0$, and integrate by parts in the resulting formula. This yields the inequality

$$
\begin{aligned}
& \frac{\nu}{2} \int_{\hat{\omega}_{2}(0)}\left|v_{y^{\prime} y}\right|^{2} \phi d y+\int_{\hat{\omega}_{2}(0)} \hat{a}^{\alpha \beta}(y) H_{y_{\beta}} \phi_{y_{\alpha}} d y+T_{\epsilon}+M_{\epsilon} \\
& \leq \int_{\hat{\omega}_{2}(0)}\left(g(y) \phi+G^{\alpha}(y) \phi_{y_{\alpha}}\right) d y
\end{aligned}
$$

where $\nu=\nu_{1} \nu_{2},\left|v_{y^{\prime} y}\right|^{2}=\sum_{\tau<n-1, \alpha<n} v_{y_{\tau} y_{\alpha}}^{2}, T_{\epsilon}$ and $M_{\epsilon}$ are integrals with penalty function to be estimated separately, and $g$ and $G^{\alpha}$ are certain functions bounded on $\widehat{\omega}_{r_{0}}(0)$ and admitting the estimate

$$
|g(y)|+|G(y)| \leq c\left|v_{y}\right|^{2}, \quad y \in \widehat{\omega}_{r_{0}}(0),
$$

in accordance with the second condition in (49). Here $v=v^{\epsilon}(y)$.

Note also that

$$
\int_{\hat{\omega}_{2}(0)}\left(\left|v_{y}\right|^{2}+\tilde{\chi}_{\epsilon}\right) d y=f_{\omega_{2 / \sqrt{e_{0}}\left(x^{0}\right)}}\left(\left|u_{x}^{\epsilon}\right|^{2}+\chi_{\epsilon}\right) d x \underset{(37)}{<} c \theta^{2}
$$

because $2 / \sqrt{e_{0}}<\rho_{0}$ by (52).

Now, we have

$$
\begin{aligned}
& T_{\epsilon}=\int_{\hat{\omega}_{2}(0)}\left[\widetilde{\chi}_{\epsilon}^{\prime} d d_{k}^{\prime}\right]_{y_{\tau}}^{\prime} v_{y_{\tau}}^{k} \phi d y \\
&=\int_{\hat{\omega}_{2}(0)}\left\{\widetilde{\chi}_{\epsilon}^{\prime \prime} 2 d^{2}\left(d^{\prime}, v_{y_{\tau}}\right)^{2} \phi d y+\tilde{\chi}_{\epsilon}^{\prime}\left(d^{\prime}, v_{y_{\tau}}\right)^{2} \phi+\tilde{\chi}_{\epsilon}^{\prime} d d_{k m}^{\prime \prime} v_{y_{\tau}}^{m} v_{y_{\tau}}^{k} \phi\right\} d y \\
& \geq-c \int_{\hat{\omega}_{2}(0)} \tilde{\chi}_{\epsilon}\left|v_{y_{\tau}}\right|^{2} \phi d y-c \int_{\hat{\omega}_{2}(0)}\left(\widetilde{\chi}_{\epsilon}^{\prime} d\right)\left|v_{y_{\tau}}\right|^{2} \phi d y \\
& \underset{(15),(6)}{\geq}-c \int_{\hat{\omega}_{2}}\left|v_{y_{\tau}}\right|^{2} \phi d y-c \int_{\hat{\omega}_{2}}\left(\tilde{\chi}_{\epsilon}^{\prime} d\right)\left|v_{y_{\tau}}\right|^{2} \phi d y .
\end{aligned}
$$

The integral $M_{\epsilon}$ is estimated similarly:

$$
\begin{aligned}
M_{\epsilon} & =\int_{\hat{\gamma}_{2}(0)}\left[\hat{\chi}_{\epsilon}^{\prime} d d_{k}^{\prime}\right]_{y_{\tau}}^{\prime} v_{y_{\tau}}^{k} \phi d \gamma \\
& \geq-c \int_{\hat{\gamma}_{2}(0)} \hat{\chi}_{\epsilon}\left|v_{y_{\tau}}\right|^{2} \phi d \gamma-c \int_{\hat{\gamma}_{(0)}}\left(\hat{\chi}_{\epsilon}^{\prime} d\right)\left|v_{y_{\tau}}\right|^{2} \phi d \gamma .
\end{aligned}
$$

The boundary condition for $v=v^{\epsilon}$ looks like this:

$$
\widehat{b}_{k l}(y, v) v_{y_{n}}^{l}=\hat{\chi}_{\epsilon}^{\prime} d d_{k}^{\prime}, \quad k \leq N, \quad y \in \widehat{\gamma}_{r_{0}}(0) .
$$

By the boundary condition, we have $\hat{b}_{k l}(y, v) v_{y_{n}}^{l} d_{k}^{\prime}=\hat{\chi}_{\epsilon}^{\prime} d$, and

$$
\sup _{\hat{\gamma}_{r_{0}}(0)}\left(\hat{\chi}_{\epsilon}^{\prime} d\right) \leq \mu \sup _{\hat{\gamma}_{r_{0}}(0)}\left|v_{y_{n}}\right| \leq \underset{(49)}{\leq} c .
$$


Next, since $\frac{2}{\sqrt{e_{0}}}<\rho_{0}$ by (52), it follows that

$$
\int_{\hat{\gamma}_{2}(0)} \hat{\chi}_{\epsilon} d \hat{\gamma}=f_{\gamma_{2 / \sqrt{e_{0}}}\left(x^{0}\right)} \chi_{\epsilon} d \gamma 2^{n-2} \underset{(37)}{\leq} c_{5} \theta^{2}
$$

We fix $y^{0} \in \widehat{\gamma}_{2}(0)$ by the condition

$$
\hat{\chi}_{\epsilon}\left(d^{2}\left(v\left(y^{0}\right)\right)\right) \leq \frac{c_{5} \theta^{2}}{\left|\widehat{\gamma}_{2}\right|}=c_{6} \theta^{2} .
$$

Then for every $y \in \widehat{\gamma}_{2}(0)$ we have

$$
\hat{\chi}_{\epsilon}\left(d^{2}(v(y))\right) \leq c_{6} \theta^{2}+\sup _{\hat{\omega}_{2}} \sum_{\tau \leq n-1}\left|\left(\hat{\chi}_{\epsilon}\right)_{y_{\tau}}^{\prime}\right|\left|y_{\tau}-y_{\tau}^{0}\right| \underset{(59)}{\leq} c_{6} \theta^{2}+c=c_{7} .
$$

Therefore,

$$
\sup _{\hat{\gamma}_{2}(0)} \hat{\chi}^{\epsilon}\left(d^{2}(v(y))\right) \leq c_{7} .
$$

Now we can assert that

$$
\begin{aligned}
& M_{\epsilon} \underset{(59),(61)}{\geq-c} \int_{\hat{\gamma}_{2}(0)}\left|v_{y_{\tau}}\right|^{2} \phi d \gamma=c \int_{\hat{\omega}_{2}(0)}\left(\left|v_{y_{\tau}}\right|^{2} \phi\right)_{y_{n}} d y \\
& \quad=c \int_{\hat{\omega}_{2}(0)}\left(2 v_{y_{\tau}} v_{y_{\tau} y_{n}} \phi+\left|v_{y_{\tau}}\right|^{2} \phi_{y_{n}}\right) d y .
\end{aligned}
$$

The resulting volume integral admits an easy estimate and does not change the structure of inequality (55).

Next, taking the estimates for $T_{\epsilon}$ and $M_{\epsilon}$ into account, we deduce the following inequality from (55):

$$
\begin{aligned}
& \frac{\nu}{4} \int_{\hat{\omega}_{2}(0)}\left|v_{y^{\prime} y}\right|^{2} \phi d y+\int_{\hat{\omega}_{(0)}} \hat{a}^{\alpha \beta}(y) H_{y_{\beta}} \phi_{y_{\alpha}} d y \\
& \quad \leq \int_{\hat{\omega}_{2}(0)}\left(g \phi+G^{\alpha} \phi_{y_{\alpha}}\right) d y+c \int_{\hat{\omega}_{2}}\left(\tilde{\chi}_{\epsilon}^{\prime} d\right)\left|v_{y_{\tau}}\right|^{2} \phi d y .
\end{aligned}
$$

We need to estimate the last integral in (62). For this, we observe that $\tilde{\chi}_{\epsilon}$ obeys the inequality

$$
\mathcal{L}\left[\frac{\tilde{\chi}_{\epsilon}}{2}\right]+\frac{\nu}{2}\left(\widetilde{\chi}_{\epsilon}^{\prime} d\right)^{2} \leq c\left(\left|v_{y}\right|^{4}+\left|v_{y}\right|^{2}\right)
$$

a.e. on $\widehat{\omega}_{2}(0)$, where $\mathcal{L}[w] \equiv-\left(\widehat{a}^{\alpha \beta}(y) w_{y_{\beta}}\right)_{y_{\alpha}}$. Indeed, direct calculations show that

$$
\begin{aligned}
\left(\hat{a}^{\alpha \beta}\left(\frac{\tilde{\chi}_{\epsilon}}{2}\right)_{y_{\beta}}\right)_{y_{\alpha}}= & \left(\widehat{a}^{\alpha \beta} v_{y_{\beta}}^{k}\right)_{y_{\alpha}} \tilde{\chi}_{\epsilon}^{\prime} d d_{k}^{\prime} \\
& +\widehat{a}^{\alpha \beta}\left[\tilde{\chi}_{\epsilon}^{\prime \prime} d^{2}\left(d^{\prime}, v_{y_{\alpha}}\right)\left(d_{y_{\beta}}^{\prime}\right)+\tilde{\chi}_{\epsilon}^{\prime}\left(d^{\prime}, v_{y_{\alpha}}\right)\left(d^{\prime}, v_{y_{\beta}}\right)+\left(\tilde{\chi}_{\epsilon}^{\prime} d\right) d_{k m}^{\prime \prime} v_{y_{\beta}}^{k} v_{y_{\alpha}}^{m}\right] ;
\end{aligned}
$$

we can find $\left(\hat{a}^{\alpha \beta} v_{y_{\beta}}^{k}\right)_{y_{\alpha}}$ from the system for the function $v^{\epsilon}$ and plug the result into the inequality obtained above. This leads to the relation

$$
\mathcal{L}\left[\frac{\tilde{\chi}_{\epsilon}}{2}\right]+\hat{b}^{k m} \frac{d \tilde{\chi}_{\epsilon}}{d v^{m}} \frac{d \tilde{\chi}_{\epsilon}}{d v^{k}} \leq c\left|v_{y}\right|^{2}\left|\frac{d \tilde{\chi}_{\epsilon}}{d v}\right|+c\left|v_{y}\right|^{2}
$$

a.e. in $\widehat{\omega}_{2}(0) ;\left\{\hat{b}^{k l}\right\}=\hat{b}^{-1}$ is the matrix inverse to $\hat{b}$. Inequality (63) follows.

Now, (63) gives rise to the integral inequality

$$
\begin{array}{r}
\int_{\hat{\omega}_{2}(0)} \widehat{a}^{\alpha \beta}\left(\frac{\tilde{\chi}_{\epsilon}}{2}\right)_{y_{\beta}} \phi_{y_{\alpha}}+\frac{\nu}{2} \int_{\hat{\omega}_{2}}\left(\widetilde{\chi}_{\epsilon}^{\prime} d\right)^{2} \phi d y \leq c \int_{\hat{\omega}_{2}(0)} g \phi d y, \\
\phi \geq 0, \quad \operatorname{spt} \phi \subset \hat{\omega} \cup \hat{\gamma}_{2},
\end{array}
$$


where $g$ is a bounded function satisfying (56). We have dropped the integral over $\widehat{\gamma_{2}}$ arising from integration by parts because it is nonnegative by the boundary condition (58). From (62) and (64) it follows that the scalar function $z(y)=H[v](y)+\frac{1}{2} \tilde{\chi}_{\epsilon}\left(d^{2}(v(y))\right)$, $v=v^{\epsilon}$, satisfies the inequality

$$
\begin{aligned}
& \frac{\nu}{4} \int_{\widehat{\omega}_{2}}\left(\left|v_{y^{\prime} y}\right|^{2}+\left(\widetilde{\chi}_{\epsilon}^{\prime} d\right)^{2}\right) \phi d y+\int_{\widehat{\omega}_{2}(0)} \hat{a}^{\alpha \beta} z_{y_{\beta}} \phi_{y_{\alpha}} d y \\
& \quad \leq c \int_{\hat{\omega}_{2}}\left(\tilde{\chi}_{\epsilon}^{\prime} d\right)\left|v_{y^{\prime}}\right|^{2} \phi d y+\int_{\widehat{\omega}_{2}(0)}\left(g \phi+G^{\alpha} \phi_{y_{\alpha}}\right) d y
\end{aligned}
$$

Estimating the first integral on the right by the Cauchy inequality, we see that

$$
\begin{aligned}
& \frac{\nu}{8} \int_{\widehat{\omega}_{2}(0)}\left(\left|v_{y^{\prime} y}\right|^{2}+\left(\widetilde{\chi}_{\epsilon}^{\prime} d\right)^{2}\right) \phi d y+\int_{\hat{\omega}_{2}} \widehat{a}^{\alpha \beta} z_{y_{\beta}} \phi_{y_{\alpha}} d y \\
& \leq \int_{\widehat{\omega}_{2}}\left(g \phi+G^{\alpha} \phi_{y_{\alpha}}\right) d y, \quad \phi \geq 0,\left.\quad \phi\right|_{\partial^{\prime} \widehat{\omega}_{2}(0)}=0
\end{aligned}
$$

with some functions $g$ and $G^{\alpha}$ satisfying (56), and $\partial^{\prime} \widehat{\omega}_{2}=\partial \widehat{\omega}_{2} \backslash \widehat{\gamma}_{2}$.

In (65), we put $\phi=\xi^{2}, \xi \equiv 1$ for $y \in \widehat{\omega}_{3 / 2}(0)$, spt $\xi \subset \widehat{\omega}_{2} \cup \widehat{\gamma}_{2},\left|\xi_{y}\right| \leq c$. By (56), (57), and (49), we see that

$$
\int_{\widehat{\omega}_{3 / 2}(0)}\left|v_{y^{\prime} y}\right|^{2} d y+\int_{\widehat{\omega}_{3 / 2}(0)}\left(\tilde{\chi}_{\epsilon}^{\prime} d\right)^{2} d y \leq c \theta^{2} .
$$

The nonnegative function $z$ satisfies the inequality

$$
\int_{\widehat{\omega}_{2}(0)} \widehat{a}^{\alpha \beta} z_{y_{\beta}} \phi_{y_{\alpha}} d y \leq \int_{\widehat{\omega}_{2}(0)}\left(g \phi+G^{\alpha} \phi_{y_{\alpha}}\right) d y, \quad \phi \geq 0,\left.\quad \phi\right|_{\partial^{\prime} \widehat{\omega}_{2}}=0,
$$

and moreover,

$$
\int_{\widehat{\omega}_{1}(0)} z^{2} d y \underset{(49)}{\leq} c \int_{\widehat{\omega}_{1}}\left(\left|v_{y}\right|^{2}+\tilde{\chi}_{\epsilon}\right) d y \underset{(57)}{\leq} c_{8} \theta^{2}
$$

We show that, under condition (68), inequality (67) implies that

$$
\sup _{\hat{\omega}_{1 / 2}} z(y) \leq \lambda_{1}(\theta)
$$

with some function $\lambda_{1}(\theta)$ that tends to 0 as $\theta \rightarrow 0$. To this end, we put $\phi(y)=(z(y)-$ $k)_{+} \xi^{2}(y)$ in (67), where $(z-k)_{+}=\max \{z-k, 0\}, k \geq 0$, and $\xi$ is a cutoff function for $B_{\rho}(0)(\rho \in(1 / 2,1])$ such that $\xi=1$ in $B_{\rho(1-\sigma)}(0),\left|\xi_{y}\right| \leq \frac{c}{\rho \sigma}, \rho(1-\sigma) \geq \frac{1}{2}$. As a result, we obtain

$$
\int_{A_{k, \rho}}\left|z_{y}\right|^{2} \xi^{2} d y \leq c_{9}\left(\frac{1}{(\sigma \rho)^{2}} \int_{A_{k, \rho}}(z-k)_{+}^{2} d y+\left|A_{k, \rho}\right|\right), \quad k \geq 0,
$$

where $A_{k, \rho}=\left\{y \in \widehat{\omega}_{\rho}(0): z(y)>k\right\}$.

By the lemma at the end of this section, under condition (68), inequalities (70) imply the estimate

$$
\sup _{\hat{\omega}_{1 / 2}(0)} z(y) \leq 2 k_{0}=c \theta^{\frac{2}{n+2}} \equiv \lambda_{1}(\theta)
$$

provided that $\theta \leq \theta_{1}, \theta_{1}=\theta_{1}\left(c_{9}, n\right)$.

Thus, we have obtained the first estimate in (54). To obtain the second, we put $h=(\eta)_{y_{n}}$ in (47), where spt $\eta \subset \widehat{\omega}_{r_{0}} \cup \widehat{\gamma}_{r_{0}}$, and integrate by parts in the first and the 
third summand. We arrive at

$$
\begin{aligned}
\int_{\hat{\omega}_{r_{0}}(0)} & {\left[\widehat{a}^{\alpha \beta} \widehat{b}_{k l} v_{y_{n} y_{\beta}}^{l} \eta_{y_{\alpha}}+\left[\widehat{a}^{\alpha \beta} \widehat{b}_{k l}\right]_{y_{n}}^{\prime} v_{y_{\beta}}^{l} \eta_{y_{\alpha}}-\frac{1}{2} \widehat{a}^{\alpha \beta}\left(\widehat{b}_{m l}\right)_{v^{k}}^{\prime} v_{y_{\beta}}^{l} v_{y_{\alpha}}^{m} \eta_{y_{n}}\right] d y } \\
+ & \frac{1}{2} \int_{\hat{\omega}_{r_{0}}(0)}\left(\frac{d \tilde{\chi}_{\epsilon}}{d v^{k}}\right)_{y_{n}}^{\prime} \eta d y+\frac{1}{2} \int_{\widehat{\gamma}_{r_{0}}} \frac{d \tilde{\chi}_{\epsilon}}{d v^{k}} \eta d y+\int_{\widehat{\gamma}_{r_{0}}} \hat{a}^{\tau \mu} \widehat{b}_{k l} v_{y_{\mu}}^{l} \eta_{y_{\tau}} d \gamma=0 .
\end{aligned}
$$

Now, we specify $\eta=v_{y_{n}} \phi$ in (71), where $\phi$ is a $\mathcal{C}^{1}$-smooth nonnegative function and $\operatorname{spt} \phi \subset \widehat{\omega}_{2}(0) \cup \hat{\gamma}_{2}(0)$. We observe that, in the resulting identity, the first integral over $\hat{\gamma}_{2}$ is nonnegative by the boundary condition. Also, the second integral over $\hat{\gamma}_{2}$ is well defined under the above choice of $\eta$, because the boundary condition yields an expression for $v_{y_{n}}^{k}$ that can be differentiated in the tangent directions (i.e., $v_{y_{n}} y_{\tau}$ exists on $\widehat{\gamma}_{2}$ ). This results in an inequality for the function $Q=(1 / 2) b_{k l} v_{y_{n}}^{l} v_{y_{n}}^{k}$ :

$$
\begin{gathered}
\frac{\nu}{2} \int_{\hat{\omega}_{2}}\left|v_{y_{n} y}\right|^{2} \phi d y+\int_{\hat{\omega}_{2}} \hat{a}^{\alpha \beta} Q_{y_{\beta}} \phi_{y_{\alpha}} d y+\int_{\hat{\gamma}_{2}} \hat{a}^{\tau \mu} \widehat{b}_{k l} v_{y_{\mu}}^{l}\left(v_{y_{n}}^{k} \phi\right)_{y_{\tau}} d y+D_{\epsilon} \\
\leq \int_{\hat{\omega}_{2}}\left(g \phi+G^{\alpha} \phi_{y_{\alpha}}\right) d y, \quad \phi \geq 0, \quad \operatorname{spt} \phi \subset \widehat{\omega}_{2} \cup \widehat{\gamma}_{2} .
\end{gathered}
$$

Here $D_{\epsilon}=\frac{1}{2} \int_{\hat{\omega}_{2}}\left[\frac{d \tilde{\chi}_{\epsilon}}{d v^{k}}\right]_{y_{n}}^{\prime} v_{y_{n}}^{k} \phi d y,\left|v_{y_{n} y}\right|=\left|\left(v_{y_{n}}\right)_{y}\right|$, and the bounded functions $g$ and $G^{\alpha}$ satisfy (56).

Since $D_{\epsilon} \underset{(6),(15),(49)}{\geq}-c \int_{\hat{\omega}_{2}}\left|v_{y_{n}}\right|^{2} \phi d y-c \int_{\hat{\omega}_{2}}\left(\tilde{\chi}_{\epsilon}^{\prime} d\right)\left|v_{y_{n}}\right|^{2} \phi d y$, we can deduce from (72) that

$$
\begin{aligned}
& \frac{\nu}{2} \int_{\hat{\omega}_{2}}\left|v_{y_{n} y}\right|^{2} \phi d y+\int_{\hat{\omega}_{2}} \widehat{a}^{\alpha \beta} Q_{y_{\beta}} \phi_{y_{\alpha}} d y+\int_{\hat{\gamma}_{2}} \hat{a}^{\tau \mu} \widehat{b}_{k l} v_{y_{\mu}}^{l}\left(v_{y_{n}}^{k} \phi\right)_{y_{\tau}} d \gamma \\
& \quad \leq \int_{\hat{\omega}_{2}}\left(\widetilde{\chi}_{\epsilon}^{\prime} d\right)\left|v_{y_{n}}\right|^{2} \phi d y+\int_{\hat{\omega}_{2}}\left(g \phi+G^{\alpha} \phi_{y_{\alpha}}\right) d y, \quad \phi \geq 0, \quad \operatorname{spt} \phi \subset \widehat{\omega}_{2} \cup \widehat{\gamma}_{2} .
\end{aligned}
$$

Adding (64) and (73), we easily obtain the following inequality for the function $M(y)=$ $Q(y)+\frac{\tilde{\chi}_{\epsilon}\left(d^{2}(v(y))\right.}{2}, v=v^{\epsilon}(y)$ :

$$
\begin{aligned}
& \frac{\nu}{4} \int_{\hat{\omega}_{2}}\left(\left|v_{y_{n} y}\right|^{2}+\left(\widetilde{\chi}_{\epsilon}^{\prime} d\right)^{2}\right) \phi d y+\int_{\hat{\omega}_{2}} \widehat{a}^{\alpha \beta} M_{y_{\beta}} \phi_{y_{\alpha}} d y+\int_{\hat{\gamma}_{2}} \widehat{a}^{\tau \mu} \widehat{b}_{k l} v_{y_{\mu}}^{l}\left(v_{y_{n}}^{k} \phi\right)_{y_{\tau}} d \gamma \\
& \quad \leq \int_{\hat{\omega}_{2}}\left(g \phi+G^{\alpha} \phi_{y_{\alpha}}\right) d y .
\end{aligned}
$$

Here $g, G^{\alpha}$, and $\phi$ possess the same properties as in (73).

In the last inequality, we denote the integral over $\widehat{\gamma}_{2}$ by $J$ and represent it as follows by using the boundary condition (58):

$$
\begin{aligned}
J & =\int_{\hat{\gamma}_{2}} \hat{a}^{\tau \mu}\left(\frac{d \hat{\chi}_{\epsilon}}{d v^{l}}\right)_{y_{\tau}}^{\prime} v_{y_{\mu}}^{l} \phi d \gamma-\int_{\hat{\gamma}_{2}} \hat{a}^{\tau \mu}\left[\widehat{b}_{k l}\right]_{y_{\tau}}^{\prime} v_{y_{\mu}}^{l} v_{y_{n}}^{k} \phi d \gamma+\int_{\hat{\gamma}_{2}} \widehat{a}^{\tau \mu} \widehat{b}_{k l} v_{y_{\mu}}^{l} v_{y_{n}}^{k} \phi_{y_{\tau}} d \gamma \\
& =j_{1}+j_{2}+j_{3} .
\end{aligned}
$$

Inequalities (6), (15), (49), (59), and (61) help us in obtaining the estimate

$$
\begin{aligned}
j_{1}+j_{2} & \geq-c \int_{\hat{\gamma}_{2}}\left|v_{y^{\prime}}\right|^{2} \phi d \gamma=c \int_{\hat{\omega}_{2}}\left(\left|v_{y^{\prime}}\right|^{2} \phi\right)_{y_{n}} d y \\
& \geq-\frac{\nu}{4} \int_{\hat{\omega}_{2}}\left|v_{y_{n} y}\right|^{2} \phi d y-c \int_{\hat{\omega}_{2}}\left|v_{y^{\prime}}\right|^{2} \phi d y+c \int_{\hat{\omega}_{2}}\left|v_{y^{\prime}}\right|^{2} \phi_{y_{n}} d y .
\end{aligned}
$$


Now, from (74) we deduce the inequality

$$
\begin{aligned}
\frac{\nu}{8} \int_{\hat{\omega}_{2}}\left(\left|v_{y_{n}}\right|^{2}\right. & \left.+\left(\tilde{\chi}_{\epsilon}^{\prime} d\right)^{2}\right) \phi d y+\int_{\hat{\omega}_{2}} \hat{a}^{\alpha \beta} M_{y_{\beta}} \phi_{y_{\alpha}} d y+\int_{\hat{\gamma}_{2}} \widehat{a}^{\tau \mu} \widehat{b}_{k l} v_{y_{\mu}}^{l} v_{y_{n}}^{k} \phi_{y_{\tau}} d \gamma \\
& \leq \int_{\hat{\omega}_{2}}\left(G^{\alpha} \phi_{y_{\alpha}}+g \phi\right) d y, \quad \phi \geq 0, \quad \operatorname{spt} \phi \subset \widehat{\omega}_{2} \cup \hat{\gamma}_{2},
\end{aligned}
$$

with some new functions $g$ and $G^{\alpha}$ still satisfying (56). We also observe that

$$
\int_{\hat{\omega}_{3 / 2}(0)}\left|v_{y_{n} y_{n}}\right|^{2} d y \leq c \theta^{2}
$$

to see this, it suffices to express $v_{y_{n} y_{n}}$ by using the system and to refer to (66).

Now, we turn to (75) with $\phi=(M(y)-k)_{+} \xi^{2}(y)$, where $k \geq 0$ and $\xi$ is a cutoff function for the balls $B_{\rho}(0), \rho \in(1 / 2,1]$, having the same properties as the function $\xi$ in (70). This yields

$$
\int_{A_{k, \rho}}\left|M_{y}\right|^{2} \xi^{2} d y+J_{\rho} \leq c\left\{\frac{1}{(\sigma \rho)^{2}} \int_{A_{k, \rho}}(M(y)-k)_{+}^{2} d y+\left|A_{k, \rho}\right|\right\},
$$

where the surface integral

$$
\begin{aligned}
J_{\rho} & =\int_{l_{k, \rho}} \widehat{a}^{\tau \mu} \widehat{b}_{k l} v_{y_{\mu}}^{l} v_{y_{n}}^{k}\left(M_{y_{\tau}} \xi^{2}+(M-k)_{+} 2 \xi \xi_{y_{\tau}}\right) d \gamma, \\
l_{k, \rho} & =\left\{y \in \hat{\gamma}_{\rho}: M(y)>k\right\}
\end{aligned}
$$

will be estimated separately. Differentiating the boundary condition (58) in the tangential directions, we see that

$$
\begin{aligned}
M_{y_{\tau}} & =Q_{y_{\tau}}+\frac{1}{2}\left(\tilde{\chi}_{\epsilon}\right)_{y_{\tau}}=b_{i j} v_{y_{n}}^{j} y_{\tau} v_{y_{n}}^{i}+\frac{1}{2}\left[b_{i j}\right]_{y_{\tau}} v_{y_{n}}^{i} v_{y_{n}}^{j}+\frac{1}{2}\left(\tilde{\chi}_{\epsilon}\right)_{y_{\tau}} \\
& =\left(\left(\hat{\chi}_{\epsilon}^{\prime} d d_{i}^{\prime}\right)_{y_{\tau}}-\left[b_{i j}\right]_{y_{\tau}} v_{y_{n}}^{j}\right) v_{y_{n}}^{i}+\frac{1}{2}\left[b_{i j}\right]_{y_{\tau}} v_{y_{n}}^{i} v_{y_{n}}^{j}+\frac{1}{2}\left(\tilde{\chi}_{\epsilon}\right)_{y_{\tau}} \\
& =\left(\hat{\chi}_{\epsilon}^{\prime} d d_{i}^{\prime}\right)_{y_{\tau}} v_{y_{n}}^{i}-\frac{1}{2}\left[b_{i j}\right]_{y_{\tau}} v_{y_{n}}^{i} v_{y_{n}}^{j}+\frac{1}{2}\left(\tilde{\chi}_{\epsilon}\right)_{y_{\tau}} .
\end{aligned}
$$

Now, using (15), (49), (59), (61), and the fact that the matrix $\hat{b}$ is symmetric, we can estimate the integral

$$
j_{1}=\int_{l_{k, \rho}} \widehat{a}^{\tau \mu} \widehat{b}_{k l} v_{y_{\mu}}^{l} v_{y_{n}}^{k} M_{y_{\tau}} \xi^{2} d \gamma
$$

as follows: $j_{1} \geq-c\left|l_{k, \rho}\right|$

The integral $j_{2}=\int_{l_{k, \rho}} \widehat{a}^{\tau \mu} \widehat{b}_{k l} v_{y_{\mu}}^{l} v_{y_{n}}^{k}(M-k)_{+} 2 \xi \xi_{y_{\tau}} d \gamma$ admits the estimate

$$
\left|j_{2}\right| \leq \frac{c}{\sigma \rho} \int_{l_{k, \rho}}(M-k)_{+} d \gamma \leq \frac{c}{(\sigma \rho)^{2}} \int_{l_{k, \rho}}(M-k)_{+}^{2} d \gamma+c\left|l_{k, \rho}\right| .
$$

Thus,

$$
J_{\rho}=j_{1}+j_{2} \geq-\frac{c}{(\sigma \rho)^{2}} \int_{l_{k, \rho}}(M-k)_{+}^{2} d \gamma-c\left|l_{k, \rho}\right|,
$$

and by (77) we obtain

$$
\begin{aligned}
\int_{A_{k, \rho}}\left|M_{y}\right|^{2} \xi^{2} d y \leq & c_{10}\left\{\frac{1}{(\sigma \rho)^{2}} \int_{A_{k, \rho}}(M(y)-k)_{+}^{2} d y+\left|A_{k, \rho}\right|\right\} \\
& +c_{11}\left\{\frac{1}{(\sigma \rho)^{2}} \int_{l_{k, \rho}}(M(y)-k)_{+}^{2} d \gamma+\left|l_{k, \rho}\right|\right\}, \quad k>0 .
\end{aligned}
$$


We observe that, moreover,

$$
\int_{\widehat{\omega}_{1}} M^{2} d y \leq c \theta^{2}, \quad \int_{\widehat{\gamma}_{1}} M^{2} d \gamma \leq c \theta^{2} .
$$

Indeed, the first estimate is an immediate consequence of (49) and (57). In order to verify the second estimate in (79), we introduce a function $\eta$ such that $\eta=1$ in $\widehat{\omega}_{1}(0)$ and spt $\eta \subset \widehat{\omega}_{3 / 2} \cup \widehat{\gamma}_{3 / 2}$. Then

$$
\begin{aligned}
\int_{\hat{\gamma}_{1}}\left|v_{y}\right|^{2} d \gamma & \leq \int_{\hat{\gamma}_{3 / 2}}\left|v_{y}\right|^{2} \eta d \gamma=\left|\int_{\hat{\omega}_{3 / 2}}\left(\left|v_{y}\right|^{2} \eta\right)_{y_{n}} d y\right| \\
& \leq c \int_{\hat{\omega}_{2}}\left(\left|v_{y y}\right|^{2}+\left|v_{y}\right|^{2}\right) d y \underset{(57),(66),(76)}{\leq} c \theta^{2} ; \\
\int_{\hat{\gamma}_{1}} M^{2} d \gamma & \leq c \theta^{2}+\frac{c}{e_{0}} \int_{\hat{\gamma}_{1}} \hat{\chi}_{\epsilon}^{2} d \gamma \underset{(60)}{\leq} c \theta^{2} .
\end{aligned}
$$

By the lemma at the end of this section, under condition (79) with $\theta \leq \theta_{2}=$ $\theta_{2}\left(c_{10}, c_{11}, n\right)$, inequalities (78) imply that

$$
\sup _{\hat{\omega}_{1 / 2}(0)} M(y) \leq c \theta^{\frac{n}{(n+1)(n-1)}}=\lambda_{2}(\theta) \rightarrow 0, \quad \theta \rightarrow 0 .
$$

Thus, we have deduced the second estimate in (54). As has already been mentioned, if $\theta$ is sufficiently small, then estimates (54) contradict (52). It should be noted that the choice of $\theta$ does not depend on $\epsilon \leq \epsilon_{*}$. Thus, we have obtained the first estimate in (43).

To deduce the second estimate in (43), we put $h=\left(u_{x_{\tau}} \xi^{2}\right)_{x_{\tau}}$ in (41), where $u=u^{\epsilon}$, $\tau \leq n-1$, and $\xi$ is a cutoff function for $B_{R_{5}}$ with $\xi=1$ in $B_{R_{6}}(0), R_{6}=(1 / 2) R_{5}$. After integration by parts, using the first estimate in (43) and the boundary condition (40), we obtain

$$
\begin{aligned}
& \int_{\Gamma} \chi_{\epsilon}^{\prime}\left(d^{\prime}, u_{x_{\tau}}\right)^{2} \xi^{2} d \Gamma+\int_{B^{+}} \chi_{\epsilon}^{\prime}\left(d^{\prime}, u_{x_{\tau}}\right)^{2} \xi^{2} d x+\int_{B^{+}}\left|u_{x x \prime}\right|^{2} d x \\
& \quad \leq c\left(1+\int_{B^{+}}\left(\chi_{\epsilon}^{\prime} d\right)^{2} \xi^{2} d x\right), \quad u=u^{\epsilon}, \quad B^{+}=B_{R_{5}}^{+}, \quad \Gamma=\Gamma_{R_{5}} .
\end{aligned}
$$

Direct inspection shows that the function $\chi_{\epsilon}=\chi_{\epsilon}\left(d^{2}\left(u^{\epsilon}\right)\right)$ satisfies the inequality

$$
\begin{array}{r}
-\left(a^{\alpha \beta}(x)\left(\chi_{\epsilon}\right)_{x_{\beta}}\right)_{x_{\alpha}}+\frac{1}{2} b^{m l} \frac{d \chi_{\epsilon}}{d u^{l}} \frac{d \chi_{\epsilon}}{d u^{m}}+a^{\alpha \beta}\left(d^{\prime} u_{x_{\beta}}\right)\left(d^{\prime} u_{x_{\alpha}}\right) \chi_{\epsilon}^{\prime} \leq c\left(1+\left|\frac{d \chi \epsilon}{d u}\right|\right), \\
\left\{b^{m l}\right\}=b^{-1} .
\end{array}
$$

To deduce (81), we need to express $\left(a^{\alpha \beta} u_{x_{\beta}}^{k}\right)_{x_{\alpha}}$ by using the system (40). Now, (81) implies the following integral inequality:

$$
\begin{gathered}
\int_{B^{+}} a^{\alpha \beta}\left(\chi_{\epsilon}\right)_{x_{\beta}} \phi_{x_{\alpha}} d x+\frac{\nu}{2} \int_{B^{+}}\left|\frac{d \chi_{\epsilon}}{d u}\right|^{2} \phi d x+\int_{\Gamma}\left(\chi_{\epsilon}\right)_{x_{n}}^{\prime} \phi d \Gamma \\
\leq c \int_{B^{+}}\left(1+\left|\frac{d \chi_{\epsilon}}{d u}\right|\right) \phi d x,\left.\quad \phi\right|_{S^{+}}=0, \quad \phi \geq 0 .
\end{gathered}
$$

By the boundary condition (40), the integral over $\Gamma$ in (82) is nonnegative. Putting $\phi=\left|\frac{d \chi_{\epsilon}}{d u}\right|^{m-2} \xi^{m}, m \geq 2$, where $\xi$ has the same properties as in (80), we see that

$$
\int_{B_{R_{6}}^{+}}\left(\chi_{\epsilon}^{\prime} d\right)^{m} d x \leq \int_{B^{+}}\left|\frac{d \chi_{\epsilon}}{d u}\right|^{m} \xi^{m} d x \leq c(m), \quad m \geq 2 .
$$

In particular, putting $m=2$ in (83) and invoking (800), we deduce that $\int_{B^{+}}\left|u_{x x^{\prime}}^{\epsilon}\right|^{2} d x \leq c$. Then we express $\left|u_{x_{n} x_{n}}^{\epsilon}\right|$ by using (40), obtaining the second estimate in (43). This completes the proof of Proposition 4. 
Remark 5. Since by the first estimate in (43) we have

$$
\frac{\sup }{B_{R_{5}}^{+}} \chi_{\epsilon}\left(d^{2}\left(u^{\epsilon}\right)\right) \leq c\left(\theta^{-1}, R_{5}^{-1}\right), \quad \epsilon \leq \epsilon_{*},
$$

it follows that

$$
\frac{\sup }{B_{R_{5}}^{+}} \chi\left(d^{2}\left(u^{\epsilon}\right)\right) \leq c \epsilon<\delta_{0}^{2}, \quad \epsilon \leq \epsilon_{*},
$$

if $\epsilon_{*}$ is sufficiently small ( $\delta_{0}$ is taken from condition $\left[\mathcal{A}_{\mathcal{K}}\right]$ ). By the definition of $\chi(\cdot)$, this means that $\chi\left(d^{2}\left(u^{\epsilon}\right)\right)=d^{2}\left(u^{\epsilon}\right), \epsilon \leq \epsilon_{*}, x \in \overline{B_{R_{5}}^{+}}$. In particular, the boundary condition (40) takes the form

$$
b_{k l}\left(x, u^{\epsilon}\right)\left(u^{\epsilon}\right)_{x_{n}}^{l}=\frac{d}{\epsilon} d_{u_{k}}^{\prime}\left(u^{\epsilon}\right), \quad k \leq N, \quad x \in \Gamma_{R_{5}} .
$$

In what follows, we denote $\Omega_{r}=B_{r} \cap\left\{y_{n}>-\delta\right\}$ and $\gamma_{r}=B_{r} \cap\left\{y_{n}=-\delta\right\}$, where $r \in(0,1]$ and $\delta$ is a fixed number in $[0,1]$.

Lemma. Suppose a nonnegative function $w \in W_{2}^{1}\left(\Omega_{1}\right)$ satisfies the inequalities

$$
\begin{aligned}
\int_{A_{k, \rho-\sigma \rho}}\left|w_{y}\right|^{2} d y \leq & m_{1}\left\{\frac{1}{(\sigma \rho)^{2}} \int_{A_{k, \rho}}(w-k)+^{2} d y+\left|A_{k, \rho}\right|\right\} \\
& +m_{2}\left\{\frac{1}{(\sigma \rho)^{2}} \int_{l_{k, \rho}}(w-k)_{+}^{2} d \gamma+\left|l_{k, \rho}\right|\right\}, \quad k>0,
\end{aligned}
$$

where $\rho \in(1 / 2,1), \sigma \in(0,1), A_{k, \rho}=\left\{y \in \Omega_{\rho}: w(y)>k\right\}, l_{k, \rho}=\left\{y \in \gamma_{r}: w(y)>k\right\}$, $k>0$.

Then there exists a constant $\tau_{*}=\tau_{*}\left(m_{1}, m_{2}, n\right)<1$ with the property that if

$$
\|w\|_{2, \Omega_{1}}+\|w\|_{2, \gamma_{1}} \leq \tau
$$

for some $\tau \leq \tau_{*}$, then

$$
\sup _{\Omega_{1 / 2}} w \leq 2\left(\frac{\tau}{\tau_{*}}\right)^{\frac{n}{(n+2)(n-1)}} .
$$

If in [a] we have $m_{2}=0$ and $\|w\|_{2, \Omega_{1}} \leq \tau$ for $\tau \leq \tau_{*}$, then instead of [b] we obtain

$$
\sup _{\Omega_{1 / 2}} w \leq 2\left(\frac{\tau}{\tau_{*}}\right)^{\frac{2}{n+2}} \text {. }
$$

\section{§6. An estimate of the Hölder NORM FOR the GRAdient of $u(x)$}

We prove the following result for the solution $u(x)$ of the variational problem (13).

Proposition 5. There exist positive constants $\theta$ and $R_{2}$ such that if (12) is fulfilled with these parameters, then the solution $u$ of problem (13) belongs to $\mathcal{C}^{1, \beta}\left(\overline{B_{\tau R_{2}}^{+}}\right)$and

$$
\left\|u_{x}\right\|_{\mathcal{C}^{\beta}\left(\overline{B_{\tau R_{2}}^{+}}\right)} \leq c
$$

with some $\tau, \beta \in(0,1 / 2)$. The constant $c$ in (85) depends on $\beta$ and the parameters in conditions $\left[\mathcal{A}_{\mathcal{K}}\right],\left[\mathcal{A}_{1}\right]$, and $\left[\mathcal{A}_{3}\right]$.

In essence, the proof of Proposition 5 is a modification of an idea employed by Ural'tseva in the study of the regularity for the Signiorini problem; see 39. 
Proof. Let $u$ be a solution of problem (13), and let (12) be fulfilled with $\theta$ and $R_{2}$ taken from Proposition 4. Then the approximate solutions $u^{\epsilon}$ obey (25) and (37); moreover, $\chi\left(d^{2}\left(u^{\epsilon}\right)\right)=d^{2}\left(u^{\epsilon}\right), \epsilon \leq \epsilon_{*}$. The function $v=u_{x_{\tau}}^{\epsilon}, \tau \leq n-1$, satisfies the identity

$$
\begin{aligned}
& \int_{B_{R_{6}}^{+}} a^{\alpha \beta} b_{k l} v_{x_{\beta}}^{l} \eta_{x_{\alpha}}^{k} d x+\int_{\Gamma_{R_{6}}} \frac{1}{\epsilon}\left(d^{\prime}, v\right)\left(d^{\prime}, \eta\right) d \Gamma+\int_{\Gamma_{R_{6}}} \frac{d}{\epsilon} d_{k m}^{\prime \prime} v^{m} \eta^{k} d \Gamma \\
&+\int_{B_{R_{6}}^{+}} \frac{1}{\epsilon}\left(d^{\prime}, v\right)\left(d^{\prime}, \eta\right) d x+\int_{B_{R_{6}}^{+}} \frac{d}{\epsilon} d_{k m}^{\prime \prime} v^{m} \eta^{k} d x \\
&=\int_{B_{R_{6}}^{+}}\left[\Phi^{\alpha} \eta_{x_{\alpha}}+Q \eta\right] d x, \quad \eta \in W_{2}^{1}\left(B_{R_{6}}^{+}\right),\left.\quad \eta\right|_{S_{R_{6}}^{+}}=0 .
\end{aligned}
$$

Here $\Phi^{\alpha}$ and $Q$ are certain functions bounded on $B_{R_{6}}^{+}$.

Let $M=\max \frac{}{B_{R_{6}}^{+}}\left|u^{\epsilon}(x)\right|$, and let $\mathbb{S}_{M}=\partial \mathcal{K} \cap \mathbf{B}_{M}(0)$ be a compact part of the surface $\partial \mathcal{K}$. Let $\mathbf{B}_{M}(0)=\left\{u \in \mathbb{R}^{N}:|u|<M\right\}$. There exists $\delta_{1}>0$ such that the smooth projection $w_{P}=P \operatorname{Pr}_{\mathbb{S}_{M}} w, w \in V_{\delta_{1}}\left(\mathbb{S}_{M}\right)$, is well defined on a two-sided neighborhood $V_{\delta_{1}}\left(\mathbb{S}_{M}\right)$.

Clearly, $u(x) \in \mathcal{K} \cap \mathbf{B}_{M}(0)$ for $x \in \overline{B_{R_{6}}^{+}}$. Two cases are possible: 1$) \operatorname{dist}\left(u(0), \mathbb{S}_{M}\right) \leq$ $\frac{1}{2} \delta_{1}$, or 2$) \operatorname{dist}\left(u(0), \mathbb{S}_{M}\right)>\frac{1}{2} \delta_{1}$. In the second case, $\operatorname{dist}\left(u^{\epsilon}, \mathbb{S}_{M}\right)>0$ and $u^{\epsilon} \in \operatorname{int} \mathcal{K}$ for $\epsilon \leq \epsilon_{*}$ and $x \in \overline{B_{R_{6}}^{+}}$provided that $\epsilon_{*}$ and $R_{2}$ are chosen sufficiently small. In this case, the problem for $u^{\epsilon}$ takes the form

$$
L u^{\epsilon}=0, \quad x \in B_{R_{6}}^{+} ;\left.\quad u_{x_{n}}^{\epsilon}\right|_{\Gamma_{R_{6}}}=0
$$

Using the split structure (5) of the matrix $A$, we can view each component $\left(u^{\epsilon}\right)^{k}, k=$ $1, \ldots, N$, as a solution of a scalar equation with zero Neumann condition on the "plane" part of the boundary:

$$
-\left(a^{\alpha \beta}(x)\left(u^{\epsilon}\right)_{x_{\beta}}^{k}\right)_{x_{\alpha}}=Z^{k}(x), \quad x \in B_{R_{6}}^{+},\left.\quad\left(u^{\epsilon}\right)_{x_{n}}^{k}\right|_{\Gamma_{R_{6}}}=0,
$$

where the entries of the matrix $a$ belong to $\mathcal{C}^{1}\left(\overline{B_{R_{6}}^{+}}\right)$and $Z^{k} \in L_{m}\left(B_{R_{6}}^{+}\right)$for $m>n$; moreover, the norms $\left\|Z^{k}\right\|_{m, B_{R_{6}}^{+}}$are bounded uniformly in $\epsilon \leq \epsilon_{*}$ (see (43), (83)). By classical results, we have

$$
\left\|\left(u^{\epsilon}\right)^{k}\right\|_{\mathcal{C}^{1, \beta}\left(\overline{B_{R_{7}}^{+}}\right)} \leq c, \quad k \leq N
$$

with $\beta=1-\frac{n}{m}, \epsilon \leq \epsilon_{*}, R_{7}=(1 / 2) R_{6}$. This proves Proposition 5 in the case in question.

We turn to the first case, which is more substantial. Clearly, in this situation the projection $u_{P}^{\epsilon}(x)$ is well defined for $x \in \overline{B_{R_{6}}^{+}}$and $\epsilon \leq \epsilon_{*}$ if $\epsilon_{*}$ and $R_{2}$ are fixed sufficiently small.

We introduce a smooth moving coordinate system

$$
\left(\lambda_{1}(w), \ldots, \lambda_{N-1}, \nu(w)\right), \quad w \in \mathbb{S}_{M}
$$

where the $\lambda_{j}(w), j \leq N-1$, belong to the tangent plane $T_{w}(\partial \mathcal{K})$ and $\nu(w)=d^{\prime}(w)$ is a normal to $T_{w}(\partial \mathcal{K})$. We observe that

$$
\sup _{w \in V_{\delta_{1}}\left(\mathbb{S}_{M}\right)}\left(\left|\nabla_{w} \lambda(w)\right|+\left|\nabla_{w} \nu(w)\right|\right) \leq c
$$

by the assumption $\left[\mathcal{A}_{\mathcal{K}}\right]$.

By (43) and (89), we see that

$$
\frac{\sup }{B_{R_{6}}^{+}} \sum_{j \leq N-1}\left|\left(\lambda_{j}\left(u_{P}^{\epsilon}(x)\right)\right)_{x}^{\prime}\right|+\left|\left(\nu\left(u_{P}^{\epsilon}(x)\right)\right)_{x}^{\prime}\right| \leq c, \quad \epsilon \leq \epsilon * .
$$


Now we fix a number $R \leq R_{6} / 2$ and a point $\widehat{x} \in \Gamma_{R / 4}(0)$. Consider the solution $G_{\rho}^{\widehat{x}}(x)$ of the problem

$$
\begin{aligned}
- & \left(a^{\alpha \beta}(x)\left(G_{\rho}^{\widehat{x}}(x)\right)_{x_{\beta}}\right)_{x_{\alpha}}=\frac{\mathbf{I}_{\omega_{\rho}(\widehat{x})}(x)}{2\left|\omega_{\rho}\right|}, \quad x \in B_{R}^{+}(0), \\
& \left.\left(G_{\rho}^{\widehat{x}}\right)_{x_{n}}^{\prime}\right|_{\Gamma_{R}}=0,\left.\quad G_{\rho}^{\widehat{x}}\right|_{S_{R}^{+}}=0, \quad \omega_{\rho}(\widehat{x})=B_{R}^{+} \cap B_{\rho}(\widehat{x}), \quad \rho \leq R / 8 .
\end{aligned}
$$

Here $\mathbf{I}_{\omega}(x)$ is the characteristic function of a set $\omega \subset \mathbb{R}^{n}$.

We extend the functions $a^{\alpha \beta}(x)$ to $B_{R}^{-}(0)$ as follows:

$$
\begin{aligned}
& \tilde{a}^{\alpha \beta}\left(x^{\prime}, x_{n}\right)=a^{\alpha \beta}\left(x^{\prime},-x_{n}\right), \quad \alpha, \beta \leq n-1 \quad \text { or } \quad \alpha=\beta=n ; \\
& \widetilde{a}^{\alpha \beta}\left(x^{\prime}, x_{n}\right)=-a^{\alpha \beta}\left(x^{\prime}, x_{n}\right), \quad \alpha \leq n-1, \beta=n .
\end{aligned}
$$

Then the even extension of the function $G_{\rho}^{\widehat{x}}$ to $B_{R}^{-}(0)$ is a regularization of the Green function for the Dirichlet problem in $B_{R}$ for the operator $L w=-\left(\widetilde{a}^{\alpha \beta}(x) w_{x_{\beta}}\right)_{x_{\alpha}}$. The properties of such functions are well studied (see [14, 36, 37]).

Next, we fix constants $l_{1}, \ldots, l_{N-1}$ such that

$$
\sup _{j \leq N-1}\left|l_{j}\right| \leq \sup _{x \in B_{R_{6}}^{+}}\left|u_{x}^{\epsilon}\right| \underset{(43)}{\leq} c_{*}, \quad \epsilon \leq \epsilon_{*}
$$

their values will be specified later.

We define the function $\widetilde{v}=v-\sum_{j=1}^{N-1} l_{j} \lambda_{j}\left(u_{P}^{\epsilon}(x)\right)\left(v=u_{x_{\tau}}^{\epsilon}\right.$, the constants $l_{j}$ depend on $\tau \leq n-1)$ and put $\eta=\widetilde{v} G_{\rho}^{\widehat{x}} \xi^{2}$ in (86), where $\xi$ is a cutoff function for $B_{R / 2}(\widehat{x})$ such that $\xi=1$ in $B_{R / 4}(\widehat{x})$. Then

$$
\left(d^{\prime}, v\right)\left(d^{\prime}, \eta\right)=\left(d^{\prime}, v\right)\left[\left(d^{\prime}, v\right)-\sum_{j=1}^{N-1} l_{j}\left(d^{\prime}, \lambda_{j}\left(u_{P}^{\epsilon}\right)\right)\right]=\left(d^{\prime}, v\right)^{2} \geq 0, \quad x \in \overline{B_{R}^{+}} .
$$

Next, estimates (83), (43) and the boundary condition (84) imply that

$$
\left\|\frac{d}{\epsilon}\right\|_{m, B_{R_{6}}^{+}} \leq c(m), \quad m \geq 2 ; \quad\left\|\frac{d}{\epsilon}\right\|_{\infty, \Gamma_{R_{5}}} \leq c .
$$

Taking (93), (94), and the properties of $G_{\rho}^{\widehat{x}}$ into account, by summation over $\tau=$ $1, \ldots, n-1$ we obtain

$$
\begin{array}{r}
\frac{\nu}{2} \int_{\widehat{\omega}_{R}}\left|\widetilde{v}_{x}\right|^{2} G_{\rho}^{\widehat{x}}(x) \xi^{2} d x+\int_{\widehat{\omega}_{R}} a^{\alpha \beta} H_{x_{\beta}}\left(G_{\rho}^{\widehat{x}}\right)_{x_{\alpha}} \xi^{2} d x \leq \frac{c}{R^{n}} \int_{T_{R}}|\widetilde{v}|^{2} d x+c R^{\gamma}, \\
\widehat{\omega}_{R}=B_{R / 2}^{+}(\widehat{x}), \quad T_{R}=B_{R / 2}^{+}(\widehat{x}) \backslash B_{R / 4}^{+}(\widehat{x}) .
\end{array}
$$

Here $H(x)=\frac{1}{2} b_{k l}\left(x, u^{\epsilon}\right) \widetilde{v}^{l} \widetilde{v}^{k}, \gamma=\gamma(m)>0$, and we assume that $m>n$. By (43), it is possible to pass to the limit as $\epsilon \rightarrow 0$ in (95), obtaining

$$
\frac{\nu}{2} \int_{\hat{\omega}_{R}}\left|\widetilde{v}_{x}^{o}\right|^{2} G_{\rho}^{\widehat{x}} \xi^{2} d x+\int_{\hat{\omega}_{R}} a^{\alpha \beta} H_{x_{\beta}}^{0}\left(G_{\rho}^{\widehat{x}}\right)_{x_{\alpha}} \xi^{2} d x \leq \frac{c}{R^{n}} \int_{T_{R}}\left|\widetilde{v}^{o}\right|^{2} d x+c R^{\gamma},
$$

where $\widehat{\omega_{R}}, T_{R}$, and $\gamma$ are the same as in (95), $\widetilde{v}^{o}=u_{x_{\tau}}-\sum_{j=1}^{N-1} l_{j} \lambda_{j}\left(u_{P}\right)$, and $H^{0}=$ $\frac{1}{2} b_{k l}(x, u)\left(\widetilde{v}^{o}\right)^{l}\left(\widetilde{v}^{o}\right)^{k}(u$ is the solution in question of problem (13)). The integral involving $H^{0}$ can be estimated much as it was done, e.g., in [14. More precisely, we must consider the integral identity satisfied by the solution $G_{\rho}^{\widehat{x}}$ of problem (91):

$$
\int_{B_{R}^{+}} a^{\alpha \beta}(x)\left(G_{\rho}^{\widehat{x}}\right)_{x_{\beta}} \psi_{x_{\alpha}} d x=f_{\omega_{\rho}(\widehat{x})} \frac{1}{2} \psi(x) d x, \quad \psi \in W_{2}^{1}\left(B_{R}^{+}\right),\left.\quad \psi\right|_{S_{R}^{+}}=0,
$$

and put $\psi=H^{0} \xi^{2}$ in it. 
Then the integral $J=\int_{\widehat{\omega_{R}}} a^{\alpha \beta} H_{x_{\beta}}^{0}\left(G_{\rho}^{\widehat{x}}\right)_{x_{\alpha}} \xi^{2} d x$ (which we are busy with) can be estimated as follows:

$$
J=-\int_{\widehat{\omega}_{R}} a^{\alpha \beta} H^{0} 2 \xi \xi_{x_{\beta}}\left(G_{\rho}^{\widehat{x}}\right)_{x_{\alpha}}+\frac{1}{2} \int_{\widehat{\omega}_{\rho}} H^{0} \xi^{2} d x \geq-|\mathcal{L}|,
$$

where

$$
\begin{aligned}
|\mathcal{L}| & =\left|\int_{\widehat{\omega_{R}}} A^{\alpha \beta} H^{0} 2 \xi \xi_{x_{\beta}}\left(G_{\rho}^{\widehat{x}}\right)_{x_{\alpha}} d x\right| \leq \frac{c}{R} \int_{T_{R}(\widehat{x})}\left|\widetilde{v}^{o}\right|^{2}\left|\left(G_{\rho}\right)_{x}\right| \xi d x \\
& \leq \int_{T_{R}} \frac{\left|\left(G_{\rho}\right)_{x}\right|^{2}}{G_{\rho}}\left|\widetilde{v}^{o}\right|^{2} d x+\frac{c}{R^{2}} \int_{T_{R}} G_{\rho}\left|\widetilde{v}^{o}\right|^{2} d x \\
& \leq c R^{n-2} \int_{T_{R}}\left|\left(G_{\rho}\right)_{x}\right|^{2}\left|\widetilde{v}^{o}\right|^{2} d x+\frac{c}{R^{n}} \int_{T_{R}}\left|\widetilde{v}^{o}\right|^{2} d x=c\left(R^{n-2}(\mathrm{a})+(\mathrm{b})\right) .
\end{aligned}
$$

The integral (b) was already taken into account by (96), and the integral (a) is treated as follows. Suppose a function $\eta=\eta(|x-\widehat{x}|) \geq 0$ has the properties that $\eta(\tau)=0$ outside the interval $\tau \in[R / 8, R]$ and $\eta(\tau)=1$ for $\tau \in[R / 4, R / 2],\left|\eta_{x}\right| \leq \frac{c}{R}$. Putting $\psi=G_{\rho}^{\widehat{x}}\left|\widetilde{v}^{o}\right|^{2} \eta^{2}$ in (97), we obtain

$$
\int_{D_{R}(\widehat{x})} a^{\alpha \beta}\left(G_{\rho}^{\widehat{x}}\right)_{x_{\beta}}\left(G_{\rho}^{\widehat{x}}\left|\widetilde{v}^{o}\right|^{2} \eta^{2}\right)_{x_{\alpha}} d x=0
$$

whence

$$
\begin{gathered}
(\mathrm{a}) \leq c \int_{D_{R}(\widehat{x})}\left(G_{\rho}^{\widehat{x}}\right)^{2}\left|\widetilde{v}_{x}^{o}\right|^{2} \eta^{2} d x+c \int_{D_{R}(\widehat{x})}\left(G_{\rho}^{\widehat{x}}\right)^{2}\left|\eta_{x}\right|^{2}\left|\widetilde{v}^{o}\right|^{2} d x, \\
D_{R}(\widehat{x})=B_{R}^{+}(\widehat{x}) \backslash B_{R / 8}^{+}(\widehat{x}) .
\end{gathered}
$$

Thus, the expression $\mathcal{L}$ in (98) admits the following estimate:

$$
|\mathcal{L}| \leq c \int_{D_{R}(\widehat{x})} G_{\rho}^{\widehat{x}}\left|\widetilde{v}_{x}^{o}\right|^{2} d x+\frac{c}{R^{n}} \int_{D_{R}}\left|\widetilde{v}^{o}\right|^{2} d x
$$

Now, (96) and (98) imply that

$$
\int_{B_{R / 8}^{+}(\widehat{x})} G_{\rho}^{\widehat{x}}\left|\widetilde{v}_{x}^{o}\right|^{2} d x \leq c \int_{D_{R}(\widehat{x})} G_{\rho}^{\widehat{x}}\left|\widetilde{v}_{x}^{o}\right|^{2} d x+\frac{c}{R^{n}} \int_{D_{R}}\left|\widetilde{v}^{o}\right|^{2} d x+c R^{\gamma} .
$$

We observe that

$$
\int_{D_{R}}\left|\widetilde{v}^{o}\right|^{2} d x=\int_{D_{R}}\left(u_{x^{\prime}}, \nu\left(u_{P}\right)\right)^{2} d x+\sum_{j \leq N-1} \int_{D_{R}}\left(\left(u_{x^{\prime}}, \lambda_{j}\left(u_{P}\right)\right)-l_{j}\right)^{2} d x .
$$

Put $l_{j}=\left(l_{j}^{(1)}, \ldots, l_{j}^{(n-1)}\right), l_{j}^{(\tau)}=f_{D_{R}}\left(u_{x_{\tau}}, \lambda_{j}\left(u_{P}\right)\right) d x$. We estimate the last integral in (100) by using the Poincaré inequality, which results in

$$
\int_{B_{R / 8}^{+}(\widehat{x})} G_{\rho}^{\widehat{x}}\left|u_{x \prime x}\right|^{2} d x \leq \frac{c}{R^{n-2}} \int_{D_{R}}\left|u_{x^{\prime} x}\right|^{2} d x+c R^{\gamma}+\frac{c}{R^{n}} \int_{D_{R}}\left(u_{x^{\prime}}, \nu\left(u_{P}\right)\right)^{2} d x .
$$

Now, $G_{\rho}^{\widehat{x}}(x) \rightarrow G^{\widehat{x}}(x)$ a.e. in $B_{R / 8}^{+}(\widehat{x})$ as $\rho \rightarrow 0$, and the Fatou lemma shows that

$$
\int_{B_{R / 8}^{+}(\widehat{x})} G^{\widehat{x}}\left|u_{x^{\prime} x}\right|^{2} d x \leq c \int_{D_{R}} G^{\widehat{x}}\left|u_{x \prime}\right|^{2} d x+c R^{\gamma}+\frac{c}{R^{n}} \int_{D_{R}}\left(u_{x^{\prime}}, \nu\left(u_{P}\right)\right)^{2} d x .
$$

Next, we want to prove that

$$
\int_{B_{R / 8}^{+}(\widehat{x})} G^{\widehat{x}}\left|\left(u_{x_{n}}\right)_{x}\right|^{2} d x \leq c \int_{D_{R}(\widehat{x})} G^{\widehat{x}}\left|\left(u_{x_{n}}\right)_{x}\right|^{2} d x+\frac{c}{R^{n}} \int_{D_{R}}\left|u_{x_{n}}\right|^{2} d x+c R^{\gamma}
$$


where the set $D_{R}$ is as in (101). To do this, we put $h=\left(u_{x_{n}}^{\epsilon} G_{\rho}^{\widehat{x}} \xi^{2}\right)_{x_{n}}$ in (41), where $G_{\rho}^{\widehat{x}}$ and $\xi$ are as before. After integration by parts, we obtain

$$
\begin{aligned}
\int_{\hat{\omega}_{R}} & \left\{\left(A_{k l}^{\alpha \beta} u_{x_{\beta} x_{n}}^{l}+\left[A_{k l}^{\alpha \beta}\right]_{x_{n}}^{\prime} u_{x_{\beta}}^{l}\right)\left(u_{x_{n}}^{k} G_{\rho}^{\widehat{x}} \xi^{2}\right)_{x_{\alpha}}-\frac{1}{2}\left(A_{m l}^{\alpha \beta}\right)_{u^{k}}^{\prime} u_{x_{\beta}}^{l} u_{x_{\alpha}}^{m}\left(u_{x_{n}}^{k} G_{\rho}^{\widehat{x}} \xi^{2}\right)_{x_{n}}\right\} d x \\
& +\frac{1}{2} \int_{\hat{\omega}_{R}}\left(\left(\frac{d \chi_{\epsilon}}{d u}\right)_{x_{n}}^{\prime}, u_{x_{n}}\right) G_{\rho}^{\widehat{x}} \xi^{2} d x+\frac{1}{2} \int_{\hat{\gamma}_{R}}\left(\frac{d \chi_{\epsilon}}{d u}, u_{x_{n}}\right) G_{\rho}^{\widehat{x}} \xi^{2} d \gamma \\
& +\int_{\hat{\gamma}_{R}} a^{\tau \mu} b_{k l} u_{x_{\mu}}^{l}\left(u_{x_{n}}^{k} G_{\rho}^{\widehat{x}} \xi^{2}\right)_{x_{\alpha}} d \gamma=0, \quad u=u^{\epsilon}, \quad \hat{\gamma}_{R}=\Gamma_{R / 2}(\widehat{x}) .
\end{aligned}
$$

Taking (43), (94), and the properties of $G_{\rho}$ into account, we arrive at the following inequality, in which $Z(x)=\frac{1}{2} b_{k l}\left(x, u^{\epsilon}\right)\left(u^{\epsilon}\right)_{x_{n}}^{l}\left(u^{\epsilon}\right)_{x_{n}}^{k}$ :

$$
\int_{\hat{\omega}_{R}(\widehat{x})} G_{\rho}^{\widehat{x}}\left|\left(u_{x_{n}}^{\epsilon}\right)_{x}\right|^{2} \xi^{2} d x+\int_{\hat{\omega}_{R}} a^{\alpha \beta} Z_{x_{\beta}}\left(G_{\rho}^{\widehat{x}}\right)_{x_{\alpha}} \xi^{2} d x+L_{\Gamma}^{\epsilon} \leq \frac{c}{R^{n}} \int_{T_{R}}\left|u_{x_{n}}^{\epsilon}\right|^{2} d x+c R^{\gamma} .
$$

Here $\widehat{\omega}_{R}$ and $T_{R}$ have been defined earlier, and $L_{\Gamma}^{\epsilon}$ denotes the surface integral over $\Gamma_{R / 2}(\widehat{x})$. In the expression

$$
L_{\Gamma}^{\epsilon}=\int_{\Gamma_{R / 2}(\widehat{x})} a^{\tau \mu} b_{k l}\left(u^{\epsilon}\right)_{x_{\mu}}^{l}\left[\left(u^{\epsilon}\right)_{x_{n}}^{k} G_{\rho}^{\widehat{x}} \xi^{2}\right]_{x_{\tau}} d \Gamma+\int_{\Gamma_{R / 2}(\widehat{x})} \frac{d}{\epsilon}\left(d^{\prime}, u_{x_{n}}^{\epsilon}\right) G_{\rho} \xi^{2} d \Gamma,
$$

the last integral is nonnegative by the boundary condition (84). To estimate the first integral, we differentiate the boundary condition in the tangent directions $\tau=1, \ldots, n-1$ and calculate $u_{x_{n} x_{\tau}}^{\epsilon}$ on $\Gamma_{R / 2}(\widehat{x})$. As a result, we obtain the estimate

$$
L_{\Gamma}^{\epsilon} \underset{(94)}{\geq}-c R+\int_{\Gamma_{R / 2}(\widehat{x})} a^{\tau \mu} b_{k l}\left(u^{\epsilon}\right)_{x_{\tau}}^{l}\left(u^{\epsilon}\right)_{x_{n}}^{k}\left[G_{\rho}^{\widehat{x}} \xi^{2}\right]_{x_{\mu}}^{\prime} d \Gamma .
$$

The integral $j_{\epsilon}$ on the right in (104) will hamper the deduction of an integral estimate similar to (102) for the penalty function $u^{\epsilon}$; however, we observe that

$$
\begin{aligned}
\lim _{\epsilon \rightarrow 0} j_{\epsilon} & =\lim _{(84)} \int_{\Gamma_{\Gamma_{R / 2}(\widehat{x})}} a^{\tau \mu} \frac{d\left(u^{\epsilon}\right)}{\epsilon}\left(d^{\prime}\left(u^{\epsilon}\right), u_{x_{\tau}}^{\epsilon}\right)\left(G_{\rho}^{\widehat{x}} \xi^{2}\right)_{x_{\mu}} d \Gamma \\
& =\int_{\Gamma_{R / 2}(\widehat{x})} a^{\tau \mu}\left(u_{x_{\tau}}, d^{\prime}(u)\right) \psi(x) \mathbf{1}_{u \in \partial \mathcal{K}}\left[G_{\rho}^{\widehat{x}} \xi^{2}\right]_{x_{\mu}} d \Gamma \underset{(*)}{=} 0 .
\end{aligned}
$$

Indeed, the second estimate in (94) and the uniform convergence of $u^{\epsilon}$ to $u$ on $\Gamma_{R_{6}}$ guarantee the existence of a nonnegative function $\psi \in L_{\infty}\left(\Gamma_{R_{6}}\right)$ such that $d / \epsilon \rightarrow \psi \mathbf{1}_{u \in \partial \mathcal{K}}$ *-weakly in $L_{\infty}\left(\Gamma_{R_{6}}\right)$; here $\mathbf{1}_{u \in \partial \mathcal{K}}$ is the characteristic function of the set on which $u(x) \in \partial \mathcal{K}, x \in \Gamma_{R_{6}}$. Identity $(*)$ is true because $(d(u))_{x_{\tau}}^{\prime}=\left(d^{\prime}, u_{x_{\tau}}\right)=0, \tau \leq n-1$, on the set where $u \in \partial \mathcal{K}$. From (104) and (105) it follows that

$$
\lim _{\epsilon \rightarrow 0} L_{\Gamma}^{\epsilon} \geq-c R \text {. }
$$

We pass to the limit as $\epsilon \rightarrow 0$ in (103). This yields

$$
\int_{\hat{\omega}_{R}} G_{\rho}^{\widehat{x}}\left|\left(u_{x_{n}}\right)_{x}\right|^{2} \xi^{2} d x+\int_{\hat{\omega}_{R}} a^{\alpha \beta} Z_{x_{\beta}}^{o}\left(G_{\rho}^{\widehat{x}}\right)_{x_{\alpha}} \xi^{2} d x \leq \frac{c}{R^{n}} \int_{T_{R}}\left|u_{x_{n}}\right|^{2} d x+c R^{\gamma},
$$

where $Z^{0}=\frac{1}{2} b_{k l} u_{x_{n}}^{l} u_{x_{n}}^{k}$. We estimate the summand involving $Z^{0}$ with the help of (97), much as we treated the summand involving $H^{0}$ in (96). This results in the estimate

$$
\int_{B_{R / 8}^{+}(\widehat{x})} G_{\rho}^{\widehat{x}}\left|\left(u_{x_{n}}\right)_{x}\right|^{2} d x \leq c \int_{D_{R}} \frac{\left|\left(u_{x_{n}}\right)_{x}\right|^{2}}{R^{n-2}} d x+\frac{c}{R^{n}} \int_{D_{R}}\left|u_{x_{n}}\right|^{2} d x+c R^{\gamma} .
$$

In this inequality, we pass to the limit as $\rho \rightarrow 0$ and use the Fatou lemma, obtaining (102). Thus, the limit function $u$ satisfies (101) and (102). 
Next, we put $t_{R}(\widehat{x})=\Gamma_{R}(\widehat{x}) \backslash \Gamma_{R / 8}(\widehat{x})$ and

$$
t_{R}^{(1)}=\left\{x \in t_{R}(\widehat{x}): u(x) \in \partial \mathcal{K}\right\}, \quad t_{R}^{(2)}=\left\{x \in t_{R}(\widehat{x}): u(x) \in \operatorname{int} \mathcal{K}\right\} .
$$

For a fixed $R \leq R_{6} / 2$, two cases are possible: (a) $\left|t_{R}^{(1)}\right| \geq \frac{1}{2}\left|t_{R}\right|$, or (b) $\left|t_{R}^{(2)}\right| \geq \frac{1}{2}\left|t_{R}\right|$. In case (a), we have $d(u(x))=0$ on the set $t_{R}^{(1)}$ of "thick" measure. Consequently, $\left(d^{\prime}, u_{x_{\tau}}\right)=0, \tau \leq n-1$, on this set. By the Poincaré inequality, we have

$$
\int_{D_{R}}\left(u_{x^{\prime}}, \nu(u)\right)^{2} d x \leq c R^{2} \int_{D_{R}}\left|\left[\left(u_{x^{\prime}}, \nu\right)^{2}\right]_{x}^{\prime}\right|^{2} d x .
$$

Now, (101) shows that

$$
\int_{B_{R / 8}^{+}(\widehat{x})} G^{\widehat{x}}\left|u_{x^{\prime} x}\right|^{2} d x \leq c \int_{D_{R}} G^{\widehat{x}}\left|u_{x^{\prime} x}\right|^{2} d x+c R^{\gamma} .
$$

In case (b), the boundary condition $b_{k l}(x, u) u_{x_{n}}^{l}=\psi(x) \mathbf{1}_{u \in \partial \mathcal{K}} \nu^{k}(u), k \leq N$, guarantees that $u_{x_{n}}=0$ for $x \in t_{R}^{(2)}$, where $t_{R}^{(2)}$ is of large measure on $t_{R}$. Applying the Poincaré inequality, we obtain

$$
\int_{D_{R}}\left|u_{x_{n}}\right|^{2} d x \leq c R^{2} \int_{D_{R}}\left|\left(u_{x_{n}}\right)_{x}\right|^{2} d x .
$$

Now, by (102) we have

$$
\int_{B_{R / 8}^{+}} G^{\widehat{x}}\left|\left(u_{x_{n}}\right)_{x}\right|^{2} d x \leq c \int_{D_{R}} G^{\widehat{x}}\left|\left(u_{x_{n}}\right)_{x}\right|^{2} d x+c R^{\gamma} .
$$

Having obtained (107) and (108), we may further argue as in the author's paper [30]. We present the details for completeness.

Applying the "hole-filling" method, from the last two inequalities we deduce that

$$
\int_{B_{R / 8}^{+}(\widehat{x})} G^{\widehat{x}} w d x \leq q \int_{B_{R}^{+}(\widehat{x})} G^{\widehat{x}} w d x+c R^{\gamma}
$$

with a parameter $q \in(0,1)$. For every fixed $R \leq(1 / 2) R_{6}$, in (109) we have $w=\left|u_{x^{\prime} x}\right|^{2}$ or $w=\left|\left(u_{x_{n}}\right)_{x}\right|^{2}$. We write (109) in the form

$$
\psi(R / 8, w) \leq q \psi(R, w)+c R^{\gamma},
$$

where $\psi(r, w)=\int_{B_{r}^{+}} G^{\widehat{x}}(x) w(x) d x$ and $\gamma$ is fixed in $(0,1)$.

To argue by iteration, we fix a value of the radius $\rho \in(0, R)$ arbitrarily and consider the sequence $R_{j}=\frac{R}{8^{j}}, j=0, \ldots, M+1$, where $M$ is chosen from the condition

$$
R_{M+1} \leq \rho<R_{M}
$$

Next, among two options for $w$ we choose that one for which (110) is true for at least $\left[\frac{M}{2}\right]$ radii $R_{j}, j \leq M$. We denote this sequence of radii by $\widehat{R}_{s}=R_{j_{s}}, s \leq m,\left[\frac{M}{2}\right] \leq m \leq M$, and argue by iteration for the above choice of $w$ and the sequence $\left\{\widehat{R}_{s}\right\}$.

As a result, we arrive at the inequality

$$
\int_{B_{\rho}^{+}(\widehat{x})} G^{\widehat{x}}\left|u_{x^{\prime} x}\right|^{2} d x \leq c\left(\frac{\rho}{R}\right)^{\alpha}\left\{\int_{B_{R}^{+}(\widehat{x})} G^{\widehat{x}}\left|u_{x^{\prime} x}\right|^{2} d x+1\right\}
$$

or

$$
\int_{B_{\rho}^{+}(\widehat{x})} G^{\widehat{x}}\left|\left(u_{x_{n}}\right)_{x}\right|^{2} d x \leq c\left(\frac{\rho}{R}\right)^{\alpha}\left\{\int_{B_{R}^{+}(\widehat{x})} G^{\widehat{x}}\left|\left(u_{x_{n}}\right)_{x}\right|^{2} d x+1\right\},
$$

where $R \leq R_{6} / 2, \widehat{x} \in \Gamma_{R / 4}(0), \rho \leq R$, and $\alpha=\alpha(q)$ is a parameter in the interval $(0,1)$. (Since $\gamma \in(0,1)$ can be fixed arbitrarily, we may assume that $\gamma \geq \alpha$.) 
It should be noted that, analyzing the above proof, we can see that, avoiding the limit passage as $\epsilon \rightarrow 0$ in (95), we can estimate $u^{\epsilon}$ with $\epsilon \leq \epsilon_{*}$ as in (101) and, therefore, as in (111).

It can easily be checked that the expressions in braces in the last two inequalities are bounded. Now we see that, in any case, $u$ satisfies the inequality

$$
T_{\rho}(\widehat{x}) \equiv \int_{B_{\rho}^{+}(\widehat{x})} G^{\widehat{x}}\left|\left(u_{x_{n}}\right)_{x}\right|^{2} d x \leq c\left(R_{6}^{-1}\right) \rho^{\alpha},
$$

where $\widehat{x} \in \Gamma_{R / 4}(0), \rho \leq R \leq R_{6} / 2$. Indeed, either (112) is true, or we estimate $T_{\rho}(\widehat{x})$ by the invocation of system (40) (satisfied by the functions $u^{\epsilon}$ ) and by employing (94) and the fact that (111) is true both for $u$ and for $u^{\epsilon}$.

In a similar and even simpler way, we deduce an estimate of the form (113) for $\widehat{x} \in$ $B_{R / 2}^{+}(0), R \leq \min \left\{R_{6} / 2, \operatorname{dist}\left(\widehat{x}, \Gamma_{R}(0)\right)\right\}$. "Gluing" these two estimates (internal and near the boundary), we conclude that (113) is true for all $\widehat{x} \in \overline{B_{R_{7}}^{+}(0)}, R_{7}=R_{6} / 8$. This means that $u_{x_{n}} \in \mathcal{C}^{\beta}\left(\overline{B_{R_{7}}^{+}}\right), \beta=\alpha / 2$.

After that, we view each component $u^{k}$ of $u$ as the solution of the standard Neumann problem:

$$
\begin{aligned}
& -\left(a^{\alpha \beta} u_{x_{\beta}}^{k}\right)_{x_{\alpha}}=g^{k}(x), \quad x \in B_{R_{7}}^{+}, \\
& g^{k} \in L_{m}\left(B_{R_{7}}^{+}\right), \quad m<\infty,\left.\quad u_{x_{n}}^{k}\right|_{\Gamma_{R_{7}}} \in \mathcal{C}^{\beta}\left(\Gamma_{R_{7}}\right) .
\end{aligned}
$$

The theory of linear boundary-value problems shows that $u \in \mathcal{C}^{1, \beta}\left(\overline{B_{R_{8}}^{+}}\right), R_{8}=R_{7} / 2=$ $\tau R_{2}$, with some $\tau<1 / 2$. This proves Proposition 5 .

Remark 6. It should be noted that the Hölder exponent $\beta$ and the value of $\theta$ are fixed independently of the choice of $x^{0} \in \partial \Omega$. Moreover, the restrictions on $R_{2}$ in (12) that emerged in the proofs of Propositions 3, 4, and 5 are determined by the problem data and do not depend on $x^{0}$.

What has been said above allows us to fix $\theta_{0}$ and $R_{0}$ in (8) and (10) so as to ensure the claim of Theorem 3.

It is easily seen that the set $\Omega_{0}$ defined by (9) is relatively open in $\bar{\Omega}$. Thus, Theorem 3 is proved.

To complete the proof of Theorem 1, we observe that all points of $\Omega_{0}$ are points of smoothness for the solution $u(x)$ of problem (11), (2), (5). By the definition (8), it is clear that the closed set $\Sigma=\Sigma_{\theta_{0}, R_{0}}$ admits the estimate $H_{n-2}(\Sigma)=0$. This proves Theorem 1.

\section{\$7. Proof of Theorem 2}

Let $u$ be the solution of the variational problem (3)-(5), and let (10) be fulfilled in a neighborhood of some point fixed on $\partial \Omega$. (We retain the notation of the preceding sections.)

As before, we consider the model variational problem in the half-ball and construct a family of penalty problems for it, much as we did in $\S 2$. Surely, in the present case the penalty function $\chi\left(d^{2}(\cdot)\right)$ depends on the distance function $d=d(u, S)$ defined in a two-sided neighborhood of the surface $S$.

Condition $\left[\mathcal{A}_{S}\right]$ makes it possible to repeat the arguments of $\S \S 2-5$. We only mention a distinction in estimation of the Hölder norm for the gradient of $u$ in $\S 6$. In the present case, the limit function $u(x)$ takes values on $S$ for $x \in \overline{B_{R}^{+}}$and, consequently, $d(u(x))=0$, 
$\left(d^{\prime}, u_{x_{\tau}}\right)=0, \tau \leq n-1$, almost everywhere on this set. Thus, the last integral in (101) disappears. This enables us to obtain the estimate

$$
\psi(R / 8) \leq q \psi(R)+c R^{\gamma}, \quad \psi(r)=\int_{B_{r}^{+}(\widehat{x})} G^{\widehat{x}}\left|u_{x^{\prime} x}\right|^{2} d x
$$

with some $q \in(0,1)$ for every $R \leq R_{6} / 2$. Iterating, we arrive at the inequality

$$
\psi(\rho) \leq c\left(R^{-1}, \beta\right) \rho^{2 \beta}
$$

with some $\beta \in(0,1 / 2)$.

Recall that the solutions $u^{\epsilon}$ of the penalty problems satisfy (40). From (43) and (94) it follows that the limit function $u$ is the solution of the system

$$
-\left(A_{k l}^{\alpha \beta}(x, u) u_{x_{\beta}}^{l}\right)_{x_{\alpha}}+\frac{1}{2}\left(A_{m l}^{\alpha \beta}\right)_{u^{k}}^{\prime} u_{x_{\beta}}^{l} u_{x_{\alpha}}^{m}+\lambda(x) \nu^{k}(u)=0, \quad k \leq N,
$$

almost everywhere in $B_{R_{6}}^{+}$. Here $\lambda(x)$ is a certain scalar function, all finite powers of which are integrable on $B_{R_{6}}^{+}$.

System (116) and inequality (115) show that

$$
\int_{B_{\rho}^{+}(\widehat{x})} G^{\widehat{x}}\left|u_{x_{n} x_{n}}\right|^{2} d x \leq c \rho^{2 \beta}, \quad \rho \leq R_{6} / 2, \quad \widehat{x} \in \Gamma_{R_{6} / 2} .
$$

We note that the integral involving $\lambda(x)$ has been estimated with the help of the Hölder inequality with the exponents $s=\frac{n}{n-2+2 \beta}, s^{\prime}=\frac{s}{s-1}(\beta$ comes from (115)) in the following way:

$$
\int_{B_{\rho}^{+}(\widehat{x})} G^{\widehat{x}} \lambda^{2} d x \leq\left\|G^{\widehat{x}}\right\|_{s, B_{\rho}^{+}}\|\lambda\|_{2 s^{\prime}, B_{\rho}^{+}}^{2} \leq c\|\lambda\|_{2 s^{\prime}, B_{R_{6} / 2}^{+}}^{2} \rho^{2 \beta}, \quad 2 s^{\prime}=\frac{n}{1-\beta}>n .
$$

As was mentioned in $\S 6$, an estimate of the form (117) can be obtained for all $\widehat{x} \in \overline{B_{R_{7}}^{+}}$, $\rho \leq R_{7}, R_{7}=R_{6} / 8$. This ensures the relation $u_{x_{n}} \in \mathcal{C}^{1, \beta}\left(\overline{B_{R_{7}}^{+}}\right)$.

Now, viewing each component $u^{k}$ of $u$ as the solution of problem (114), we conclude that $u \in \mathcal{C}^{1, \beta}\left(\overline{B_{R_{8}}^{+}}\right), R_{8}=R_{7} / 2$. As was shown in $\S 6$, this implies the claim of Theorem 2 .

\section{REFERENCES}

[1] M. Giaquinta and E. Giusti, On the regularity of the minima of variational integrals, Acta Math. 148 (1982), 31-46. MR0666107 (84b:58034)

[2] — The singular set of the minima of certain quadratic functionals, Ann. Scuola Norm. Sup. Pisa Cl. Sci. (4) 11 (1984), 45-55. MR0752579 (86a:49086)

[3] J. Jost and M. Meier, Boundary regularity for minima of certain quadratic functionals, Math. Ann. 262 (1983), 549-561. MR.0696525 (84i:35051)

[4] M. Wiegner, Ein optimaler Regularitätssatz für schwache Lösungen gewisser elliptischer Systeme, Math. Z. 147 (1976), 21-28. MR0407430 (53:11205)

[5] S. Hildebrandt and K. O. Widman, On the Hölder continuity of weak solutions of quasilinear elliptic systems of second order, Ann. Scuola Norm. Sup. Pisa Cl. Sci. (4) 4 (1977), 145-178. MR0457936 (56:16140)

[6] F. Duzaar, Variational inequalities and harmonic mappings, J. Reine Angew. Math. 374 (1987), 39-60. MR0876220 (88m:58038)

[7] F. Duzaar and M. Fuchs, Optimal regularity theorems for variational problems with obstacles, Manuscripta Math. 56 (1986), 209-234. MR0850371 (87k:49015)

[8] M. Fuchs, A regularity theorem for energy minimizing maps of Riemannian manifolds, Comm. Partial Differential Equations 12 (1987), 1309-1321. MR0888462 (88g:58041)

[9] _ Some remarks on the boundary regularity for minima of variational problems with obstacles, Manuscripta Math. 54 (1985), 107-119. MR0808683 (87b:49050)

[10] M. Fuchs and N. Fusco, Partial regularity results for vector-valued functions which minimize certain functionals having nonquadratic growth under smooth side conditions, J. Reine Angew. Math. 390 (1988), 67-78. MR0953677(89h:49005) 
[11] M. Fuchs and M. Wiegner, The regularity of minima of variational problems with graph obstacles, Arch. Math. (Basel) 53 (1989), 75-81. MR.1005172 (90f:49004)

[12] S. Hildebrandt, Harmonic mappings of Riemannian manifolds, Harmonic Mappings and Minimal Immersions (Montecatini, 1984), Lecture Notes in Math., vol. 1161, Springer, Berlin, 1985, pp. 1117. MR0821968 (87j:58030)

[13] S. Hildebrandt, H. Kaul, and K.-O. Widman, An existence theorem for harmonic mappings of Riemannian manifolds, Acta Math. 138 (1977), 1-16. MR0433502 (55:6478)

[14] S. Hildebrandt and K.-O. Widman, Variational inequalities for vector-valued functions, J. Reine Angew. Math. 309 (1979), 191-220. MR0542048 (81a:35023)

[15] A. Arkhipova, Variational problem with an obstacle in $\mathbb{R}^{N}$ for a class of quadratic functionals, Zap. Nauchn. Sem. S.-Peterburg. Otdel. Mat. Inst. Steklov. (POMI) 362 (2008), 15-47. MR2760549

[16] H. Beir ao da Veiga and F. Conti, Equazioni ellittiche non lineari con ostacoli sottili, Ann. Scuola Norm. Sup. Pisa (3) 26 (1972), 533-562. MR0364862 (51:1116)

[17] L. Caffarelli, Further regularity for the Signorini problem, Comm. Partial Differential Equations 4 (1979), 1067-1075. MR0542512 (80i:35058)

[18] J. Frehse, On Signorini's problem and variational problems with thin obstacles, Ann. Scuola Norm. Sup. Pisa Cl. Sci. (4) 4 (1977), 343-362. MR0509085 (58:22987)

[19] D. Kinderlehrer, The smoothness of the solution of the boundary obstacle problem, J. Math. Pures Appl. (9) 60 (1981), 193-212. MR0620584 (84j:49011)

[20] N. N. Ural'tseva, A problem with one-side conditions on the boundary for a quasilinear elliptic equation, Probl. Mat. Anal., No. 6, Leningrad. Univ., Leningrad, 1977, pp. 172-189. (Russian) MR0509596 (58:23049)

[21] - On the regularity of solutions of variational inequalities, Uspekhi Mat. Nauk 42 (1987), no. 6, 151-174; English transl., Russian Math. Surveys 42 (1987), no. 6, 191-219. MR0933999 (90c:35033)

[22] I. Athanasopoulos and L. Caffarelli, Optimal regularity of lower dimensional obstacle problems, Zap. Nauchn. Sem. S.-Peterburg. Otdel. Mat. Inst. Steklov. (POMI) 310 (2004), 49-66; English transl., J. Math. Sci. (N.Y.) 132 (2006), no. 3, 274-284. MR2120184 (2006i:35053)

[23] G. Fichera, Existence theorems in elasticity, Handbuch der Physik, Bd. 6a/2, Springer-Verlag, Berlin, 1972.

[24] J. Nečas, On regularity of solutions to nonlinear variational inequalities for second order elliptic systems, Rend. Mat. (6) 8 (1975), 481-498. MR0382827 (52:3709)

[25] N. Ural'tseva, Strong solutions of the generalized Signorini problem, Sibirsk. Mat. Zh. 19 (1978), no. 5, 1204-1212; English transl., Siberian Math. J. 19 (1978), no. 5, 850-856. MR0508511 (80i:35166)

[26] R. Schumann, Zur Regularität einer Kontakt-Randwertaufgabe, Z. Anal. Anwendungen 9 (1990), no. 5, 455-465. MR1119544 (93e:73040)

[27] A. A. Arkhipova and N. N. Ural'tseva, Regularity of solutions of diagonal elliptic systems under convex constraints on the boundary of the domain, Zap. Nauchn. Sem. Leningrad. Otdel. Mat. Inst. Steklov. (LOMI) 152 (1986), 5-17; English transl., J. Soviet Math. 40 (1988), no. 5, 591-598. MR0869237 (87m:35107)

[28] Regularity of the solutions of variational inequalities with convex constraints on the boundary of the domain for nonlinear operators with a diagonal principal part, Vestnik Leningrad. Univ. Mat. Mekh. Astronom. 1987, vyp. 3, 13-19. (Russian) MR0928154 (89f:35088)

[29] Limit smoothness of the solutions of variational inequalities under convex constraints on the boundary of the domain, Zap. Nauchn. Sem. Leningrad. Otdel. Mat. Inst. Steklov. (LOMI) 163 (1987), 5-16; English transl., J. Soviet Math. 49 (1990), no. 5, 1121-1128. MR0918937(88m:49001)

[30] A. Arkhipova, Signorini-type problem in $\mathbb{R}^{N}$ for a class of quadratic functionals, Nonlinear Partial Differential Equations and Related Topics, Amer. Math. Soc. Transl. (2), vol. 229, Amer. Math. Soc., Providence, RI, 2010, pp. 15-38. MR2667630

[31] M. Giaquinta, Multiple integrals in the calculus of variations and nonlinear elliptic systems, Ann. of Math. Stud., vol. 105, Princeton Univ. Press, Princeton, NJ, 1983. MR0717034 (86b:49003)

[32] O. A. Ladyzhenskaya and N. N. Ural'tseva, Linear and quasilinear equations of elliptic type, 2nd ed., Nauka, Moscow, 1973; English transl. of 1st ed., Acad. Press, New York-London, 1968. MR0509265 (58:23009) MR0244627 (39:5941)

[33] M. Giaquinta and E. Giusti, Nonlinear elliptic systems with quadratic growth, Manuscripta Math. 24 (1978), 323-349. MR.0481490 (58:1606) 
[34] A. Arkhipova, On the regularity of solutions of boundary-value problem for quasilinear elliptic systems with quadratic nonlinearity, Probl. Mat. Anal., No. 15, S.-Peterburg. Gos. Univ., St. Petersburg, 1995, pp. 47-69; English transl., J. Math. Sci. 80 (1996), no. 6, 2208-2225. MR1420674 (98b:35054)

[35] Y. Chen and M. Struwe, Existence and partial regularity results for the heat flow for harmonic maps, Math. Z. 201 (1989), 83-103. MR0990191 (90i:58031)

[36] K.-O. Widman, Inequalities for the Green functions of second order elliptic operators, Univ. Linköping, Inst. Math., 1972.

[37] - Inequalities for the Green function and boundary continuity of the gradient of solutions of elliptic differential equations, Math. Scand. 21 (1967), 17-37 (1968). MR0239264 (39:621)

[38] L.-C. Evans, Partial regularity for stationary harmonic maps into spheres, Arch. Rational Mech. Anal. 116 (1991), 101-113. MR.1143435 (93m:58026)

[39] N. N. Ural'tseva, Estimation on the boundary of the domain of derivatives of solutions of variational inequalities, Probl. Mat. Anal., No. 10, Leningrad. Gos. Univ., Leningrad, 1986, pp. 92-105; English transl., J. Soviet Math. 45 (1989), no. 3, 1181-1191. MR0860572 (87k:35106)

Department of Mathematics and Mechanics, St. Petersburg State University, UniversitetSkaya Ul. 28, Stary Petergof, St. Petersburg 198504, Russia

E-mail address: arina@AA1101.spb.edu

Received 7/APR/2010

Translated by S. KISLYAKOV 